\title{
Human DNA-PK activates a STING-independent DNA sensing pathway
}

Katelyn Burleigh

\begin{abstract}
A dissertation
submitted in partial fulfillment of the requirements for the degree of
\end{abstract}

Doctor of Philosophy

University of Washington

2019

Reading Committee:

Daniel B. Stetson, Chair

Joshua J. Woodward

Jason G. Smith

Program Authorized to Offer Degree:

Molecular and Cellular Biology 
(C) Copyright 2019

Katelyn Burleigh 


\title{
University of Washington
}

\author{
Abstract \\ Katelyn Burleigh \\ Chair of Supervisory Committee: \\ Daniel B. Stetson \\ Department of Immunology
}

Human DNA-PK activates a STING-independent DNA sensing pathway

Recognition of foreign nucleic acids is critical for antiviral defense. Detection of DNA is mediated by the cGAS-STING pathway, which activates a potent type I interferon response. This pathway is broadly required for antiviral defense across cell types and species, and its relevance in context of infection, cancer, and autoimmunity has been thoroughly established. However, we have discovered an additional, STING-independent DNA sensing pathway (SIDSP) in human cells. Using STING KO human cell lines, we find that the induction of interferon is indeed abrogated at early timepoints, but surprisingly, at later timepoints, we observe a robust DNAinduced interferon response. Here we identify DNA-PK as the sensor for the SIDSP and demonstrate that its kinase activity is required for the antiviral response. We show that a heat shock protein HSPA8/HSC70 is phosphorylated after DNA stimulation and acts as a marker for the SIDSP. Finally, we explore how DNA viruses antagonize both the cGAS-STING pathway and the SIDSP. Our work highlights the importance of nucleic acid sensing for both host and virus and has implications for modulating DNA sensing in order to improve therapies for cancer or autoimmunity. 


\section{Table of Contents}

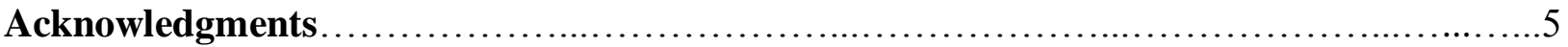

\section{Chapter 1: Introduction}

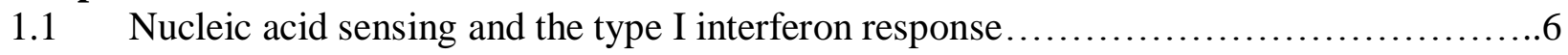

1.2 Links between the DNA Damage Response and innate immunity..................... 12

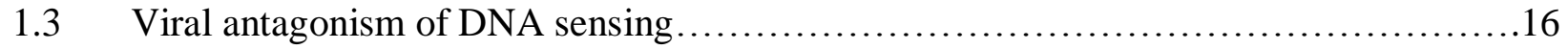

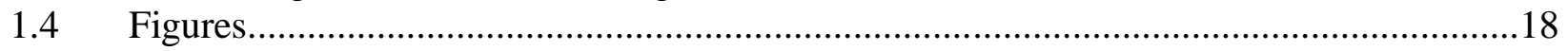

Chapter 2: E1A antagonizes two DNA sensing pathways

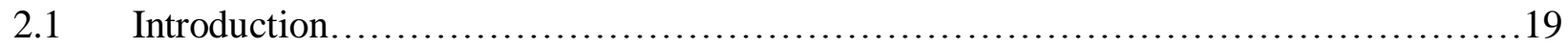

2.2 Results

E1A blocks a late step in DNA-induced IRF3 activation....................... 19

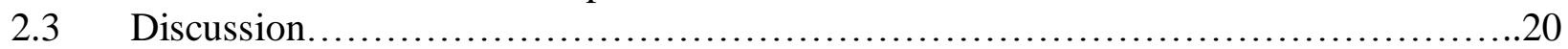

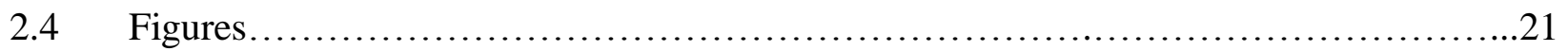

\section{Chapter 3: Characterization of a STING-independent DNA sensing pathway}

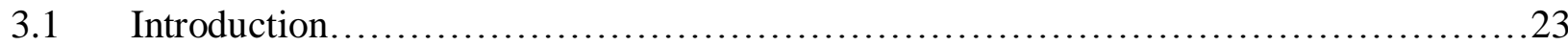

3.2 Results

STING KO human cell lines have a robust IFN response to DNA.................23

Circular plasmid DNA does not activate the SIDSP ...........................24

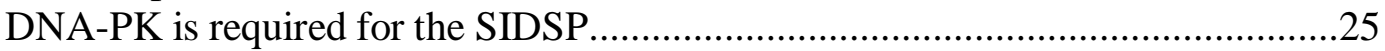

The SIDSP induces a broad antiviral response...............................26

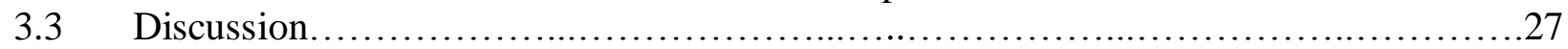

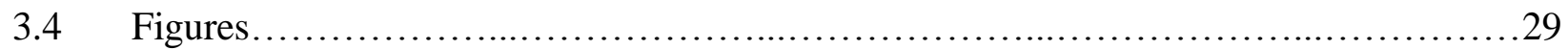

\section{Chapter 4: Identification of a specific marker of the SIDSP}

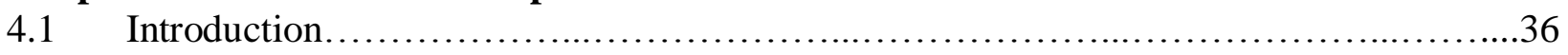

4.2 Results.

Identification of a DNA-induced phosphoprotein............................. 36

Phosphorylation of HSPA8 is conserved across species ....................................36

Phosphorylated HSPA8 is a marker for the SIDSP ............................................37

The antiviral modality of DNA-PK is distinct from its DNA damage modality....38

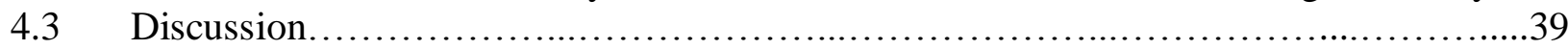

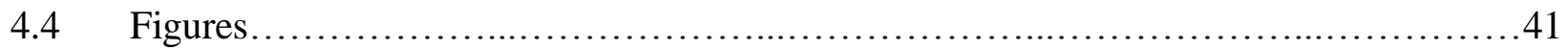

\section{Chapter 5: Conclusions}

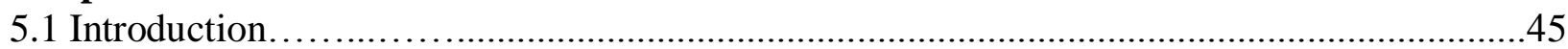

5.2 Potential relevance of the SIDSP in context of cancer and autoimmunity ...........................45

5.3 Potential relevance of the SIDSP for DNA virus infection...............................46

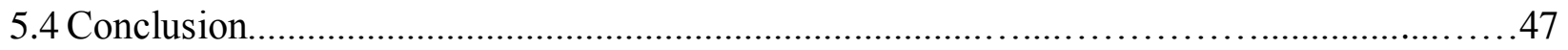

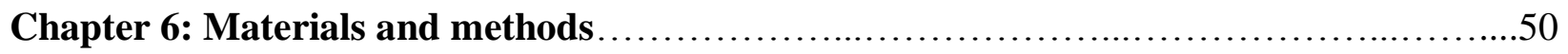

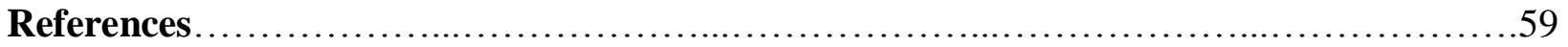




\section{Acknowledgements}

To my parents: thank you for fostering the curiosity, independence, work ethic, and determined persistence that have been absolutely required for this endeavor.

To my sister: thank you for your encouragement and world-class pep talks.

To the Weathers family: thank you for sparking my passion for biology. Nullius en verba!

To Drs. Holly Wichman and Martina Ederer: thank you for mentoring me - for patiently

teaching me in the lab, giving me opportunities to present my work, and advocating for me.

To my husband: thank you for listening to all of my presentations at the panic hour of the night, and for always believing in me. I could not have done it without you!

To my lab: thank you for the scientific discussions/comradery and especially for taking care of my experiments when my baby was sick.

To Dan: thank you for your encouragement over the years. Without your guidance and relentless optimism, I would have been quite lost. 


\section{Chapter 1: Introduction}

\subsection{Nucleic acid sensing and type I interferon}

The detection of foreign nucleic acids is central to all life. As it indicates viral infection, survival demands a rapid response. Perhaps the simplest mechanism of detection and clearance of foreign DNA is the bacterial restriction enzyme, which recognizes and cleaves specific DNA sequences present in phages. In animals from flies to humans, germline-encoded pattern recognition receptors (PRRs) bind and initiate signaling cascades that activate host defense responses. For vertebrates, this includes a robust type I interferon (IFN) response, which is the strong upregulation of IFN- $\beta$ and IFN- $\alpha$. The interferon- $\alpha / \beta$ receptor (IFNAR) is broadly expressed and its signaling results in the upregulation of hundreds of interferon stimulated genes (ISGs). These ISGs include the PRRs themselves to enhance sensing, negative regulators of signaling downstream of IFNAR, and both broad and specific antiviral effectors. IFN-treated cells are akin to fields burnt before an invading army; normal cellular functions are abrogated in order to prevent productive infection. For example, the ISG Protein Kinase R (PKR) is activated by dsRNA and leads to a block in translation, which would prevent synthesis of both cellular and viral proteins ${ }^{1}$. The importance of the type I IFN response is highlighted by the severe immunodeficiency of IFNAR KO mice; indeed, the LD50 for intravenously injected vesicular stomatitis virus (VSV) is typically $10^{8}$ in WT mice and in IFNAR KO mice, it is only 30-50 $\mathrm{pfu}^{2}$. This phenotype can be attributed not only to the central role of type I IFN in the early responses to infection, but also to the requirement for type I IFN in instructing and activating the adaptive immune response $\mathrm{e}^{3,4}$.

Nucleic acid sensors can be divided into two categories: those that survey endosomes and are generally expressed in phagocytic immune cells, and those that monitor the cytosol and are more 
broadly expressed. The endosomal sensors are part of the Toll-like receptor (TLR) family, and include TLRs 3, 7, 8, and 9. TLR3 recognizes ssRNA, TLR 7 and 8 recognize dsRNA, and TLR9 binds unmethylated CpG DNA motifs that are rare in the mammalian genome. Signaling occurs through the adaptor protein MyD88 (TLRs 7-9) or TRIF (TLR3) to activate the expression of IFNs and chemokines through the interferon regulatory factor (IRF) transcription factors. Additionally, inhibition of nuclear factor-kappa B (NF-kB) is released, allowing for proinflammatory gene expression ${ }^{5}$.

Cytosolic RNA sensing

A TLR3-independent dsRNA sensing pathway was described in 2003, where the synthetic dsRNA ligand poly I:C was shown to induce high levels of type I IFN after cytosolic delivery through lipid-based transfection or electroporation ${ }^{6}$. In contrast, this ligand is typically introduced directly to immune cells to stimulate TLR3 without transfection aid. This observation was quickly followed by the discovery of the intracellular sensors RIG-I ${ }^{7}$ and MDA5 ${ }^{8}$. Just as TLR sensors signal through adaptor proteins that create signaling platforms, the RNA sensors were found to require a mitochondrial protein, MAVS, to link RNA sensing to activation of NF$\mathrm{kB}$ and, through the kinases TBK1 and IKKi, IRF3/7-dependent gene expression ${ }^{9}$. A simplified schematic of RNA sensing is illustrated in Figure 1.

\section{Cytosolic DNA sensing}

Almost in parallel, a TLR9-independent DNA sensing pathway was first discovered in $2006^{10}$. Short 45 bp dsDNA lacking CpG sequences (termed interferon stimulatory DNA or ISD) induced a robust type I interferon response when transfected into a variety of immune cell types; this response was independent of Myd88, TRIF, and TLR9. Again, in contrast to CpG DNA, which is taken up through endocytosis, ISD required transfection. Additional data supporting the 
existence of a cytosolic DNA sensing pathway was the observation that the endosomal acidification inhibitor, chloroquine, potently blocked type I IFNs after CpG DNA, but not ISD stimulation $^{10}$. The signaling adaptor STING was discovered two years later in independent cDNA screens ${ }^{11,12}$ and was shown to be critical in mouse embryonic fibroblasts (MEFs) for the response to a variety of DNA ligands included sheared calf thymus (CT) DNA, 45 bp ISD, and transfected viral DNA. Additionally, Sting ${ }^{-/}$mice were highly susceptible to a DNA virus, herpes simplex virus (HSV-1) ${ }^{13}$. The road to the discovery of the DNA sensor that transmits signal to STING would take four more years and would be littered with controversy and confusion.

\section{Cytosolic DNA sensors}

Z-DNA binding protein 1 (ZBP1, also known as DAI) was initially a promising candidate for the elusive DNA sensor. It was shown to bind DNA in the cytosol, and siRNA knockdown in the mouse L929 line reduced the cytokine response to various DNA ligands ${ }^{14}$. However, it is highly interferon inducible, which supports a role as an effector protein, and not as the primary sensor required for inducing IFN. Additionally, its role appears to be cell-type specific; neither DAI KO MEFs, nor bone marrow-derived dendritic cells or macrophages were deficient in their DNAinduced IFN responses ${ }^{15}$. RNA polymerase III recognizes AT-rich DNA (poly dA-dT) and transcribes a RIG-I ligand, but this is independent of STING and dependent on MAVS ${ }^{16}$. DDX41 is an additional proposed sensor, as knockdown in both mouse and human cells reduces the IFN response to both DNA transfection and DNA viral infection ${ }^{17}$. However, no detailed mechanism explaining how DDX41 might activate STING has been elucidated to date.

The role of the AIM-2 like receptors (ALRs) in DNA sensing has been controversial. These PYHIN proteins possess Pyrin domains (for facilitating protein-protein interactions) and at least 
one HIN domain (for DNA binding). Activation of AIM2 by dsDNA results in ASC-mediated inflammasome formation, release of the pro-inflammatory cytokine IL-1 $\beta$ and cell death. The function of the remaining ALRs is muddy, in part because there is no clear orthology of the ALR genes among mammals. Humans possess four ALR genes, and mice possess thirteen, each with various combinations of Pyrin and HIN domains. Although both IFI16 (human) and IFI204 (mouse) are structurally similar, containing one Pyrin and two Hin domains, they are not orthologues $^{18}$. This means that the results of mouse experiments will not necessarily correlate with the reality of human biology, complicating efforts to understand these proteins. This is illustrated by the fact the genetic deletion of the entire ALR locus in mice resulted in MEFs and macrophages that were perfectly competent to respond to transfected DNA ${ }^{19}$. In addition, CRISPR disruption of IFI16 in human fibroblasts had no effect on the IFNB induced by HCMV infection, while disruption of STING abrogated the IFN response ${ }^{19}$. In contrast, siRNA targeting of IFI16 in the human monocyte THP1 cell line and of IFI204 in mouse macrophages, resulted in an approximately $50 \%$ reduction of DNA-induced $I F N B^{20}$. Suffice it to say, these contradictory studies indicate that the ALRs and IFI16 are not required for DNA sensing in all cell lines but may play the role of co-factor in some contexts.

Much needed clarity was brought to the field by both the discovery of a STING-activating second messenger molecule, cyclic GMP-AMP (cGAMP), and the sensor that synthesizes it, cyclic GMP-AMP synthase (cGAS) in $2012^{21,22}$. These reports came as a landmark duo that provided an elegant, sensible mechanism of STING activation whereby cytosolic cGAS binds DNA and produces cGAMP which binds to and activates STING. It had been shown previously that bacterial cyclic di-GMP could activate $\mathrm{STING}^{23}$, but whether there was a host-derived second messenger was an open question. It was later shown that mammalian cyclic GMP-AMP 
is linked by a unique 2'-5' phosphodiester linkage, distinguishing it from bacterial cyclic dinucleotides, which have two 3'-5'linkages ${ }^{24}$.

Those initial studies demonstrated the importance of cGAS for both human and mouse cells and in the context of both DNA transfection and DNA virus infection. Importantly, they also showed that in STING-overexpressing HEK293T cells, transfection of cGAS induced strong induction of $I F N B$, in contrast to the only modest inductions by expression of DAI, IFI16 and DDX41, thus asserting the primacy of cGAS amongst the other putative sensors ${ }^{25}$. This observation is highlighted by recent studies exploring the role of IFI16 using CRISPR-generated KO human cell lines. These studies show reduced activation of STING, TBK1, and IRF3 downstream of DNA and cGAMP in human macrophages and keratinocytes, providing more evidence that IFI16 plays some role in the cellular response to DNA ${ }^{26,27}$. However, it is worthy to note that deletion of IFI16 only reduces the response to DNA; in contrast, KO of cGAS in the same cells completely abrogates all signaling downstream of STING. Thus, cGAS has emerged as the principal DNA sensor that activates STING in any context studied thus far. Further understanding the guiding principles behind the function of the other accessory DNA sensors is of interest and will likely depend on cell type, context, and ligand.

The DNA-induced signaling cascade

The molecular details by which cGAS transmits signal resulting in the transcription of IFNB and other inflammatory molecules have been carefully deciphered and lead to some simple markers of pathway activation: phosphorylation of STING, degradation of STING, and phosphorylation of both TBK1 and IRF3. STING is localized as a butterfly-shaped dimer at the endoplasmic reticulum, and after cGAMP binding, the dimer adopts a closed conformation, where the 'tips' of the wings are $20 \AA$ closer to each other ${ }^{28}$. This activated STING acts as a 
platform for TBK1, which can auto-phosphorylate at high local concentrations and subsequently activate IRF3 ${ }^{29}$. James Chen's group discovered an elegant conserved mechanism of IRF3 activation downstream of disparate signals (LPS, DNA, and RNA) ${ }^{30}$. All of the three key adaptor proteins for these ligands (TRIF, STING, and MAVS, respectively) possess a pLxIS motif, where $\mathrm{p}$ is hydrophobic, and $\mathrm{x}$ is non-aromatic. Phosphorylation at the serine in this motif is required for IRF3 association to the adaptors and subsequent activation. For STING, this critical serine is S366 and the alanine mutant version of STING is unable to activate IRF3 ${ }^{30}$. This phosphorylation event was shown to promote de-sumoylation of STING and degradation via chaperone-mediated autophagy, whereby Hsc70 delivers proteins to the lysosome for destruction $^{31}$. Thus, activation of STING correlates with degradation, an important feature for any immune signaling pathway, lest powerful sustained immune responses initiate autoimmunity.

IRF3 activation is mediated by phosphorylation of its $\mathrm{C}$ terminus by TBK1, which includes seven serine/threonine residues that are commonly divided into a group of 2 (S385, S386) and 5 (S396, S398, S402, T404, S405). These phosphorylation events lead to IRF3 dimerization and, upon translocation to the nucleus, binding to the transcriptional co-activators CBP and p300 and activation of $I F N B$ transcription $^{32}$. Phosphorylation of two of these residues, S386 and S396, is easily measured by high quality antibodies. The relative importance of these two sites has been controversial in the literature, likely in part due to different assays used. John Hiscott's group observed that the S396D phosphomimetic mutant IRF3 is a strong activator of interferon stimulatory response element (ISRE) promoters and that the S396A mutant fails to bind to the transcriptional co-activators ${ }^{33}$. Takashi Fujita's group argued that S386 is the most important serine residue, based on the observations that S385A/S386A (2A) mutant IRF3 fails to dimerize, 
but that the S396A mutant IRF3 is perfectly competent for dimerization ${ }^{34}$. The role of S385/S386 was further clarified by James Chen's work showing that the 2A mutant associated with the adaptor proteins more strongly than the WT and failed to dimerize ${ }^{30}$, indicating that the addition of the strongly negative phosphate groups is required for IRF3 to dissociate from an adaptor. If phosphorylation of S385/S386 is necessary, but not sufficient for dimerization, a phosphomimetic 2D mutant would be repelled from the adaptor and thus fail to be fully activated/dimerize; indeed, this is exactly what is shown by both the Hiscott ${ }^{33}$ and Fujita ${ }^{34}$ research groups. The conclusion, it seems, is simply that phosphorylation at both sites is required, but that they promote different outcomes: S385/S386 phosphorylation is required for the dissociation of IRF3 from signaling platforms that must necessarily occur before dimerization, whereas S396 plays a role in promoting p300 and CBP binding in the nucleus.

\subsection{Links between the DNA Damage Response and innate immunity}

Understanding how cellular DNA is distinguished from foreign DNA has been a major question in the field. The cytosolic localization of $\mathrm{cGAS}^{21}$ leant itself to the hypothesis that self: non-self-discrimination was mediated by separation of host DNA from the sensor; however, several lines of evidence indicate that the reality is likely more complex. First, the nuclear envelope breaks down during mitosis to allow for segregation of chromosomes, exposing the 3 billion base pair genome to the cytosol. Secondly, DNA viruses, excepting poxviruses, replicate their DNA in nucleus. Yet, the cGAS-STING pathway has been shown to be integral to host defense against diverse DNA viruses, including HSV-1, adenovirus, and HIV ${ }^{35,36,25}$. These seemingly conflicting data lead to the question of whether the ligand sensed by cGAS is a nuclear replication product or perhaps a defective viral particle, accidently releasing viral genome into the cytoplasm. The biology is further complicated by the fact that growing evidence 
supports the existence of a large pool of tightly chromatin-bound nuclear cGAS ${ }^{37,38}$. Whether this nuclear cGAS can be activated, and in what contexts, is of particular interest.

Putting aside the complexities regarding cGAS localization and activation, it is clear that damaged genomic DNA does escape to the cytosol and this does correlate with inflammation via the cGAS-STING pathway. This phenomenon has been shown in context of loss-of-function mutations in DNA repair factors, acute damage, and senescence. Ataxia Telangiectasia (AT) patients have loss-of-function mutations in the DNA damage kinase ATM, and their complex phenotype includes predisposition to cancer as well as autoimmune syndromes. Their fibroblasts have increased IFN and ISG transcripts at steady state and after viral infection. In ATM-deficient mouse cells, IFN is subtly elevated compared to WT cells post-irradiation, and this is dependent on STING and not on other innate immune adaptor proteins ${ }^{39}$. Similarly, loss of function of DNA repair gene $B R C A 2$ induces IFN dependent on $\mathrm{cGAS}^{40}$. Recently, the Unterholzner research group proposed a non-canonical mode of STING activation and function downstream of acute DNA damage by etoposide treatment. They observe a cGAS-independent, IFI16-dependent stimulation of STING that primarily activates NF-kB, and less so IRF3, resulting in a cytokine profile that is skewed towards IL-6 instead of IFNs. Finally, several groups reported that the cGAS-STING pathway encourages senescence as evidenced by prolonged proliferation of cGAS KO MEFs compared to $\mathrm{WT}^{41,42}$ as well as an overall reduction in the secretion of inflammatory factors altogether referred to as the senescence-associated secretory phenotype (SASP) ${ }^{41-43}$. Senescent cells downregulate the nuclear architecture protein lamin B1, and this leads to loss of nuclear envelope integrity as well as the formation of nuclear membrane blebs containing chromatin. cGAS appears to co-localize with these micronuclei and, furthermore, siRNA disruption of lamin B1 induces low level ISG upregulation ${ }^{43}$. 
Before cGAS was discovered, one hypothesis was that perhaps DNA damage machinery was involved in sensing foreign DNA. The phosphoinositide 3-kinase (PI3K)-related kinases (PIKKs), including DNA-PK, ATM and ATR, are the masters of the DNA damage response. These proteins sense double-stranded DNA breaks (DNA-PK, ATM) or stalled replication forks (ATR) and activate signaling pathways that lead to cell cycle arrest and DNA repair, or, in cases of irreparable damage, senescence and apoptosis ${ }^{44}$. Interestingly, there have been some links between these key kinases and innate immune signaling ${ }^{45-47}$, but since 2012, most of the enthusiasm for DNA sensors has been devoted to cGAS. One study implicated MRE11, a key component of the complex that both recruits and activates ATM at double-stranded breaks, as a sensor required for the IFN response to transfected DNA in MEFs. However, the authors suggest that MRE11 is upstream of STING, but show that it is not required for the response to HSV-1, which is inconsistent with the known role of STING for the response to that virus ${ }^{45}$.

\section{DNA-PK and IRF3 activation}

Several studies have focused on DNA-PK as an activator of IRF3. Before the discovery of the DNA-sensing pathway, Dr. Peter Howley's group published research linking DNA-PK to IRF3 activation ${ }^{48}$. Specifically, they observed that DNA-PK can phosphorylate IRF3 at T135 in vitro, and that this phosphorylation occurs in vivo after Sendai virus (an RNA virus) infection with kinetics that match infection-induced DNA-PK and IRF3 association. T135 phosphorylation is thought to affect IRF3 stability; in contrast, phosphorylation at the C terminus (S386 and S396, as discussed above) is important for dimerization and activation of the IFNB promoter. The relationship between phosphorylation of $\mathrm{T} 135$ and phosphorylation at the $\mathrm{C}$ terminus is currently unknown. Moreover, whether DNA stimuli result in T135 phosphorylation has not been determined. 
In 2012, Dr. Geoffrey Smith's group demonstrated that DNA-PK deficient MEFs have a reduced IFN response to 6-hour treatment of CT-DNA. Using Prkdc ${ }^{\mathrm{SCID}}$ MEFs, which express a catalytically inactive DNA-PK ${ }^{49}$, and the DNA-PK inhibitor Nu7026, the authors conclude that DNA-PK kinase activity is not required for the response to DNA. The mechanism by which a kinase could exert its effects non-catalytically to activate STING is not clear. The full DNA-PK kinase is comprised of the DNA-PK catalytic subunit as well as the proteins Ku70 and Ku80. Interestingly, they show that Ku70 binds STING during resting conditions, but not after DNA stimulation $^{47}$, but how exactly this promotes STING activation is unclear. Additional papers from this group have shown that the poxvirus vaccinia antagonizes DNA-PK-induced chemokines with two virally encoded antagonists, $\mathrm{C} 4$ and $\mathrm{C} 16^{50,51}$. They show that in vitro, the overexpression of these viral proteins reduces phosphorylation of IRF3 induced by DNA transfection. Infection of mice with viruses that lack these key inhibitors results an increase in macrophages, neutrophils, and activated $\mathrm{T}$ cells at the site of infection, which is consistent with $\mathrm{C} 4$ and $\mathrm{C} 16$ having a role in blocking the chemokines and IFN that help attract and activate these cells. The fact that a cytoplasmically-replicating virus encodes not one, but two antagonists of DNA-PK is remarkable and suggests it must be a formidable opponent to vaccinia replication. They observe that while cGAS is known to be central for STING activation, it is unknown if DNA-PK is promoting DNA sensing through STING, or acting independently. Although DNAPK is assumed to operate upstream of STING, whether these DNA sensing antagonists block the activation of STING as measured by phosphorylation and/or degradation, was not determined.

Another group identified DNA-PK as part of a novel complex including cGAS and STING ${ }^{52}$. This complex involves a scaffold, comprised of the protein HEXIM1 and the long non-coding RNA NEAT1, that appears to associate with DNA-PK and the nuclear paraspeckle proteins, as 
well as cGAS, STING, and IRF3. The authors report that transfection of ISD into HeLa cells results in DNA-PK phosphorylation at serine 2056 and that phosphorylation of IRF3 requires HEXIM1, NEAT1, and Ku70. They conclude that further studies are required to determine whether DNA-PK kinase activity plays a role in the activation of cGAS and STING. The proteins involved in this complex are largely nuclear, and this again adds to the debate about where exactly foreign DNA is recognized within a cell - in the cytosol as per the popular model, or in the nucleus, which is sensible given the fact that all DNA viruses besides poxviruses replicate in the nucleus. These observations also raise the question of how exactly cytosolic proteins like STING and IRF3 are associated with the complex. Proximity ligation assays for components of this complex or fractionating cells before immunoprecipitation may clarify this question. Clearly, there is evidence of potential cross-talk between DNA damage and innate immune signaling, but there is not yet a satisfying model.

\subsection{Viral antagonism of DNA sensing}

In order to replicate efficiently, viruses must evade or antagonize nucleic acid sensing pathways. Host-virus conflict leaves measurable changes in the history of genes that encode the host proteins that physically interface with viruses. A high ratio of non-synonymous amino acid changes at an amino acid site compared to synonymous changes across lineages is termed "positive selection" and, not surprisingly, innate immunity genes are marked by extremely high levels of positive selection. cGAS and STING are both undergoing positive selection ${ }^{53,54}$, which predicts specific virally-encoded antagonists of these key proteins. Indeed, cGAS-STING pathway antagonists encoded by diverse viruses including HSV-1 ${ }^{55-57}$, adenovirus ${ }^{58}$, human papillomavirus $(\mathrm{HPV})^{58}$, human cytomegalovirus $(\mathrm{CMV})^{59}$, Kaposi’s sarcoma herpes virus $(\mathrm{KSHV})^{60,61,62}$, and hepatitis B $(\mathrm{HBV})^{63}$ have already been discovered, and it is likely that all 
DNA viruses encode at least one cGAS/STING antagonist. Additionally, many RNA viruses encode antagonists of the cGAS-STING pathway, likely in order to avoid cGAS-mediated recognition of mitochondrial DNA that is released after infection induces mitochondrial stress ${ }^{64}$.

A particularly potent example of viral antagonism of cGAS/STING is found in KSHV, which encodes not one, but three inhibitors of the pathway, highlighting the utility of redundancy for the virus. The first, vIRF1, especially demonstrates efficiency of function as it blocks IFN signaling by two mechanisms: 1) by blocking the association of IRF3 with the co-activators p300/CBP ${ }^{65}$, and 2) by blocking the association of STING with TBK1 and subsequent phosphorylation of STING in overexpression experiments ${ }^{66}$. The second encoded antagonist, virion component ORF52 (now named KSHV inhibitor of cGAS or KicGAS), is localized to the cytoplasm and binds both DNA and cGAS to inhibit the production of cGAMP. KicGASdeficient virus induced a modest $3 \mathrm{x}$ increase in IFNB message compared to the parental virus; however, the modest nature of this phenotype is not unexpected given the presence of multiple antagonists. Finally, the smaller, cytoplasmic isoforms of the latency-associated nuclear antigen (LANA) protein were shown to co-immunoprecipitate with cGAS in the cytosol and inhibit downstream signaling ${ }^{60}$.

Interestingly, some viruses converge on the same evasion strategy. The DNA tumor viruses adenovirus and human papillomavirus encode the oncoproteins E1 A and E7, respectively, that both potently inhibit retinoblastoma $(\mathrm{Rb})$ to modulate the cell cycle and also bind to STING to block production of IFN ${ }^{58}$. Both of these functions require the LXCXE motif of the oncoprotein, as mutation of these key residues results in loss of co-immunoprecipitation with STING ${ }^{58}$. Understanding in fine molecular detail how STING binding by E1A is translated into blockade of IFN transcription was the starting point for my work, discussed in detail in Chapter 2. 


\subsection{Figures}

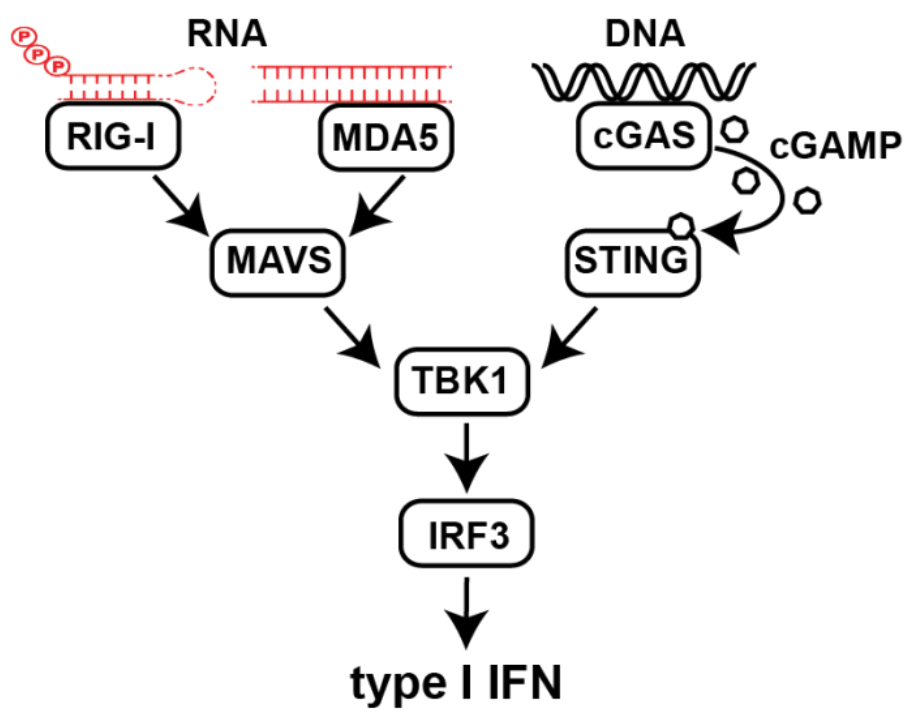

Figure 1: Cytosolic nucleic acid sensing

Foreign RNA is sensed by the RIG-I-like receptors, RIG-I and MDA5, which activate the signaling adaptor MAVS. Foreign DNA is sensed by the enzyme cGAS, which synthesizes the second messenger cGAMP to activate the signaling adaptor STING. These adaptors act as scaffolds to promote activation of the transcription factor IRF3 by the kinase TBK1.

Phosphorylated IRF3 dimerizes, translocates to the nucleus, and induces transcription of type I IFN. 


\section{Chapter 2: E1A antagonizes two DNA sensing pathways}

\subsection{Introduction}

Studying the transformation potential of E1A as well as Large T antigen (encoded by SV40)

yielded great insights into how the cell cycle is regulated through $\mathrm{Rb}^{67}$, and we hypothesized that studying the immune modulatory aspects of E1A would reveal new insights into STING signaling. In order to more fully understand how E1A binding to STING ultimately blocks IFNB transcription downstream of DNA stimulation, we characterized the phosphorylation of TBK1 and IRF3 using a HEK 293 model established previously in the lab ${ }^{58}$. HEK 293 cells were generated by transfection of sheared adenovirus genome into human embryonic kidney (HEK) cells, and the resultant transformed clonal line expresses the E1 A and E1B adenovirus proteins $^{68,69}$. These cells are deficient for an IFN response to DNA but possess intact immune responses to RNA ligands. We demonstrate that disruption of E1A expression using CRISPR is sufficient to restore the ability of DNA to induce IFN (Fig. 2A). Therefore, we sought to use this model to further understand the molecular mechanisms by which E1A blocks STING signaling.

\subsection{Results}

\section{E1A blocks a late step in DNA-induced IRF3 activation}

We discovered that comparable levels of TBK1 phosphorylation were induced by CT-DNA and RIG-I ligand in HEK 293 cells, but that phosphorylation of IRF3 S396 was markedly impaired downstream of DNA (Fig. 2B). This was rescued by E1A disruption, suggesting that E1A operates by a novel mechanism of action, somehow specifically blocking IRF3, but not TBK1, downstream of STING. To test whether these responses were truly STING dependent, we used lentiCRISPR to generate two independent clonal lines of STING KO HEK 293s and validated them by western blot and sequencing of the targeted region (Fig. 2C-D). We also 
generated a TBK1 KO line (Fig. 2E-F) as well as a control clonal line expressing the H1 nontargeting gRNA ${ }^{70}$. We expanded our analysis to include pIRF3 S386 and were surprised to see that both DNA and cGAMP induced robust phosphorylation at that site in the control HEK293 line (Fig. 2G). cGAMP induction of pIRF3 was dependent on STING and TBK1; however, surprisingly, DNA induced pIRF3 in both STING and TBK1 KO cells. Disruption of E1A restored both STING-dependent IRF3 S396 phosphorylation in response to cGAMP and STINGindependent IRF3 S396 phosphorylation in response to DNA (Fig. 2H).

\subsection{Discussion}

We discovered that E1A blocks IRF3 activation at a late step in activation, allowing only partial phosphorylation of the $\mathrm{C}$ terminus, and also that E1A blocks not one, but two DNA sensing pathways using this mechanism. These conclusions will be discussed in order. First, we have uncovered a unique mechanism of IRF3 antagonism, never before described in the literature. A fuller understanding of how E1A allows phosphorylation of one site but not the other would certainly improve our understanding of IRF3 activation. Crystallographic studies of these proteins in complex would perhaps provide insights. Intriguingly, expression of the IRF3 S396D phosphomimetic mutant was unable to rescue the IFN response to cGAMP, suggesting that blockade of S396 correlates with, but does not fully explain, the lack of IFN (data not shown), so there is certainly more to learn about this mechanism in the future. Second, this is the first description of innate immune detection of cytosolic DNA in a STING-independent manner, which has far-reaching implications, and is the subject of the following chapter (Chapter 3). 


\subsection{Figures}

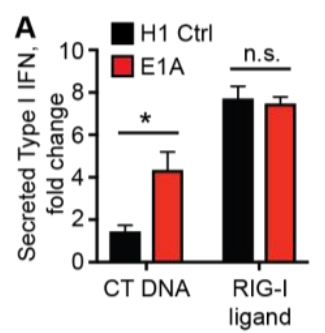

$$
\begin{aligned}
& \text { B CT RIG-I CT RIG-I }
\end{aligned}
$$

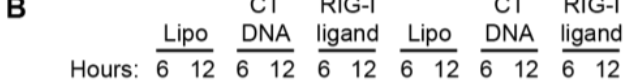

$$
\begin{aligned}
& \text { TBK1 pS172 } \\
& \text { IRF3 pS396 }-\infty-\infty
\end{aligned}
$$

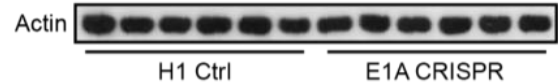

C $\varangle \infty \quad D$
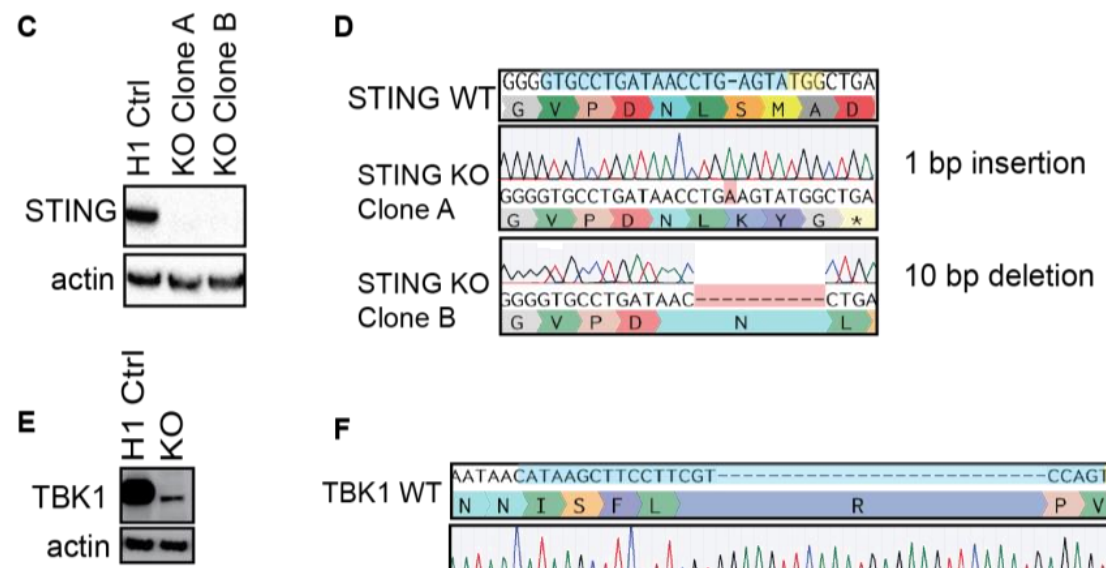

G
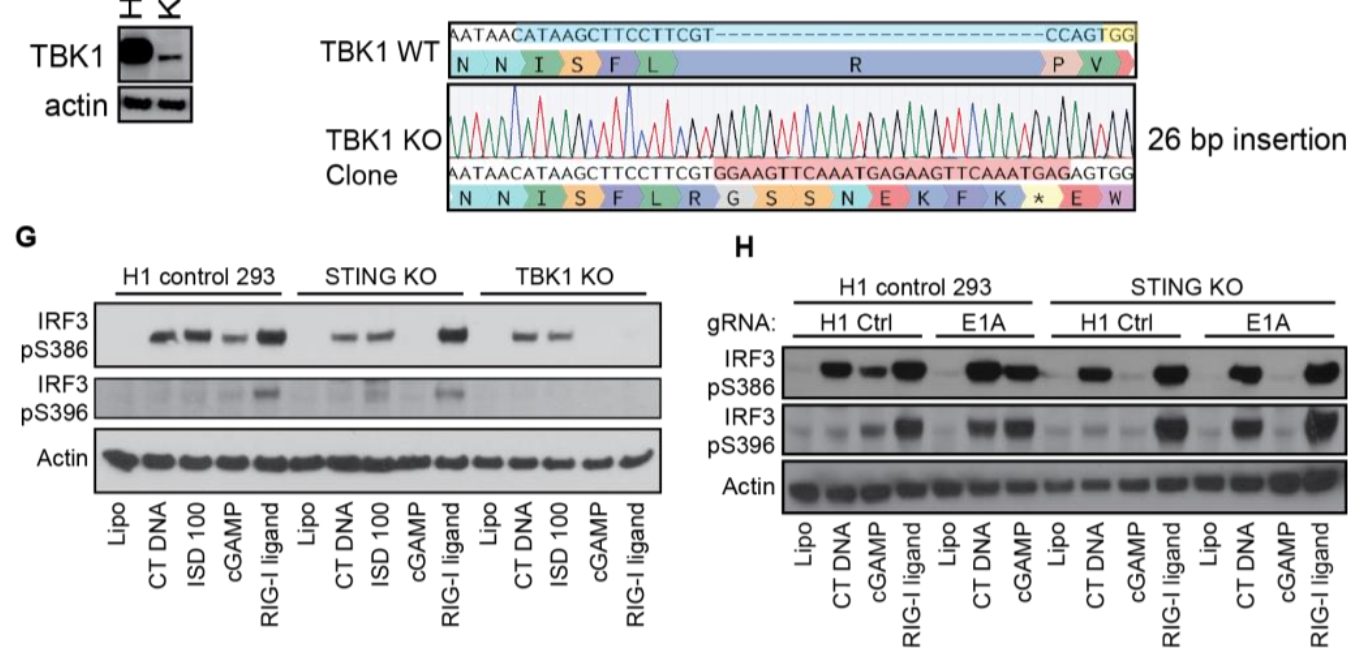

H

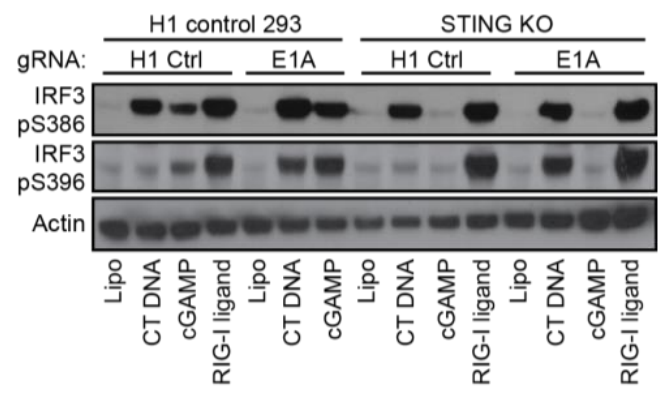

Figure 2: Human Adenovirus 5 E1A blocks two DNA sensing pathways.

(A) HEK 293 cells were transduced with lentiCRISPR constructs encoding a non-targeting H1 control or E1A-specific gRNAs, selected for three days with appropriate antibiotics, and then stimulated with CT DNA or RIG-I ligand for 12 hours, followed by measuring type I IFN activity in supernatants. $*: p<0.05$. Data is fold change relative to cells treated with Lipofectamine 2000 alone.

(B) HEK 293 cells were transfected with the indicated ligands using Lipofectamine 2000 and lysed at the indicated times post transfection, followed by western blot analysis for phosphorylated TBK1 and IRF3.

(C) HEK 293 clonal lines (H1 non-targeting control and STING KO) were generated using lentiCRISPR and assessed for STING expression by western blot. 
(D) STING KO HEK 293 clonal lines were genotyped by Sanger sequencing. The blue highlight in the WT sequence indicates the guide site and the yellow indicates the PAM. Expected cutting is $3 \mathrm{bp} 5$ ' of the PAM.

(E) HEK 293 clonal lines (H1 non-targeting control and TBK1 KO) were generated using lentiCRISPR and assessed for TBK1 expression by western blot.

(F) The TBK1 KO HEK 293 clonal line was genotyped by Sanger sequencing. The blue highlight in the WT sequence indicates the guide site and the yellow indicates the PAM. (G) HEK 293 cells and clonal lines of STING KO and TBK1 KO cells were treated with the indicated ligands for 3 hours, followed by western blot for the indicated phosphorylation sites on IRF3.

(H) HEK 293 cells and STING KO cells were transduced with lentiCRISPR contstructs as described in (A), then stimulated with the indicated ligands for 3 hours followed by western blot analysis. 


\section{Chapter 3: Characterization of a STING-independent DNA sensing pathway}

\subsection{Introduction}

Given the significance of the cGAS-STING pathway for biomedical research, a parallel DNA sensing pathway is a potentially important finding. In order to determine if the SIDSP discovered in HEK 293 cells was unique to the cell line or general to human cells, we utilized lentiCRISPR to generate additional STING KO clonal lines (Fig. 3A-B), this time in the human U937 myeloid leukemia cell line. We chose this line because 1) it was not transformed by viral oncoproteins and has intact antiviral responses to DNA and cGAMP, 2) it would be amenable to single cell cloning, and 3) being a monocyte line, it represents a different cell type than the HEK 293s. We also tested different DNA ligands to ensure the SIDSP was not specific to our sheared CT DNA ligand. This line of experiments, above and beyond confirming the generality of the SIDSP in human cells, also led us to discover the DNA sensor required for the SIDSP.

\subsection{Results}

STING KO human cell lines have a robust IFN response to DNA

Much of the initial work on cGAS and STING was done in context of mouse cells. The canonical IFN response occurs very rapidly; by 4 hours post-transfection of DNA, mouse embryonic fibroblasts (MEFs) upregulate $I f n b$ by $>1000 \mathrm{x}$, and this is dependent on STING, as is the response to cGAMP (Fig. 4A). Because the restoration of pIRF3 S396 was only measurable at 12 hours post-transfection of DNA, we assessed IFNB induction in the STING KO U937 lines at a later than usual timepoint (16 hours). Both clones failed to respond to cGAMP, but both induced IFNB1 by 500-fold in response to CT DNA, indicating the SIDSP does, in fact, exist in multiple cell lines (Fig. 4B). To better understand the kinetics of the response, we performed a time-course to compare IFNBI transcription in response to CT DNA and cGAMP in both control 
and STING KO U937s (Fig. 4C). We observed that the control cells responded to both ligands, while the STING KO U937s responded to CT DNA with a modest 50-fold increase in IFNB transcription at 4 hours post-transfection. However, the response increased over time, culminating in statistically significant 1000-fold induction of IFNBI at 16 hours. This delayed nature of the SIDSP contrasts with cGAMP-induced IFNB1, which peaks at 4-8 hours. To assess the strength of this IFN response, we measured expression of ISG15, which is induced by IFN (Fig. 4D). There was no difference in expression after 16 hours of DNA stimulation in control versus STING KO cells, suggesting that the SIDSP is a robust response. We also performed an identical time-course in MEFs and found that STING KO MEFs only upregulate Ifnb by 50-fold at any timepoint (Fig. 4E), which did not rise to the level of statistical significance. This suggests that the SIDSP is weak and difficult to measure in MEFs. Finally, to confirm that the SIDSP relies on IRF3/7 for transcription of IFNB1, we used a previously published THP1 cell line doubly deficient for these transcription factors. Indeed, the antiviral response to DNA at 16 hours was completely abrogated in this cell line (Fig. 4F).

\section{Circular plasmid DNA does not activate the SIDSP}

We tested various DNA ligands to prove the SIDSP was not a CT DNA specific artifact. Sheared herring testis DNA and synthetic $100 \mathrm{bp} \mathrm{ISD} \mathrm{also} \mathrm{activated} \mathrm{the} \mathrm{response} \mathrm{(not} \mathrm{shown).}$ However, we observed that circular plasmid DNA failed to activate STING KO cells, while still potently inducing an antiviral response in STING-sufficient control U937s (Fig. 5A). Sonication of the plasmid DNA to create a variety of linear fragments, similar to sheared CT DNA, was sufficient to restore the antiviral response in STING KO cells (Fig. 5B-C). Corroborating these data, stimulation of STING KO 293 cells with CT DNA, ISD100, and sonicated plasmid, but not 
circular plasmid, induced robust phosphorylation of IRF3 at S386 (Fig. 5D-E). These results suggest that DNA ends are required for the SIDSP in both U937 and HEK 293 cells.

\section{DNA-PK is required for the SIDSP}

There are two DNA damage kinases activated by dsDNA breaks: ATM, which is responsible for homology-directed repair (HR), and DNA-PK, which mediates non-homologous end-joining (NHEJ). To test if either were involved in the SIDSP, we pre-treated STING KO U937s with either an ATM inhibitor (KU-60019) ${ }^{71}$ or a DNA-PK inhibitor $(\mathrm{Nu} 7441)^{72}$ prior to DNA transfection and measured both IFNBI by qPCR and phosphorylation of the histone $\mathrm{H} 2 \mathrm{AX}$ on serine $139(\gamma \mathrm{H} 2 \mathrm{AX})$ by western blot. Both inhibitors reduced $\gamma \mathrm{H} 2 \mathrm{AX}$ in a dose-dependent manner, but only the DNA-PK inhibitor Nu7441 blocked the IFN response (Fig. 6A-B). Importantly, Nu7441 did not affect the RIG-I ligand-induced antiviral response, indicating that it specifically blocks the SIDSP and not IFN in general (Fig. 6C). To prove genetically that DNAPK is required for the SIDSP, we used lentiCRISPR to target STING and DNA-PK (PRKDC) simultaneously to generate a single DKO clonal line (Fig. 6D)and found that it indeed lacked an IFN response to DNA (Fig. 6E). This line grew poorly, as is consistent with other attempts to generate Prkdc-null human cell lines ${ }^{73}$.

Ku70 and Ku80 are part of the full DNA-PK kinase complex and are required for recruiting the catalytic subunit of DNA-PK to broken $\mathrm{DNA}^{44}$. In order to determine whether they are also required for the SIDSP, we attempted to generate additional DKO U937 clonal lines. However, this proved impossible as Ku70 and Ku80 are essential for human cells due to their role in telomere maintence ${ }^{74,75}$. Instead, we transiently targeted XRCC6 (Ku70) and XRCC5 (Ku80) in STING KO HEK 293s, which are more easily transduced than U937s. Immediately after selection, we plated cells for a DNA transfection experiment, and observed both loss of Ku70 
and Ku80 expression (Fig. 6F) and also loss of DNA-induced pIRF3 (Fig. 6G). Taken together, our data demonstrate pharmacologically and genetically that DNA-PK is required for the SIDSP in U937 and HEK 293 cells.

\section{The SIDSP induces a broad antiviral response}

In order to more broadly define the transcriptional response of the SIDSP, we performed global mRNA-Seq analysis of STING KO U937 cells compared to WT U937 cells treated with CT DNA, with or without $2 \mathrm{uM} \mathrm{Nu7441.} \mathrm{To} \mathrm{narrow} \mathrm{the} \mathrm{analysis,} \mathrm{we} \mathrm{first} \mathrm{focused} \mathrm{on} \mathrm{antiviral}$ genes, as defined by Gene Ontology terms. Our cutoffs for analysis were genes with a fold change of greater than 1.5 and a false discovery rate $(\mathrm{FDR})$ of $<0.05$ in any one of the comparisons. A heat map of the 124 antiviral genes that met these qualifications demonstrates that STING KO cells have a broad and potent antiviral response to CT DNA that is very similar to the response mounted by WT cells (Fig. 7A). The delayed nature of the SIDSP is confirmed and highlighted by the fact that all IFNs are more upregulated at 16 hours than at 8 hours posttransfection in STING KO U937s. In contrast, these genes plateaued or decreased in expression at 16 hours in WT cells (Fig. 7B). In fact, when comparing all upregulated genes, not just those that met the criteria for heat mapping, nearly all increased between 8 and 16 hours in the STING KO cells (Fig. 7C). This demonstrates that nearly all SIDSP-related transcriptional activity occurs later than canonical antiviral responses. Finally, we assessed the effects of Nu7441 on the DNA-induced transcriptional responses in both WT and STING KO cells. We observed only a mild effect on WT cells, which is consistent with there being two DNA sensing pathways present (Fig. 7D). However, Nu7441 markedly inhibited DNA-induced transcriptional changes in STING KO cells, with 1254/1327 (94.5\%) genes calculated as susceptible (Fig. 7E). In fact, the most strongly differentially-expressed genes after DNA stimulus were also the most sensitive to 
Nu7441, which indicates that DNA-PK is responsible for almost all of the transcriptional changes caused by CT DNA transfection in STING KO cells (Fig. 7F).

\subsection{Discussion}

Here we demonstrate the existence of a second DNA sensing pathway in human cells. Not only do we observe a strong STING-independent induction of IFNB1, but by mRNA-seq, we see a complete, robust antiviral response that overlaps with the transcriptional response induced by CT DNA in WT cells. There are three unique features of the SIDSP that distinguish it from the STING-mediated response to DNA. First, it is a delayed response, peaking roughly 8 hours later than the STING-dependent response to cGAMP. Second, it appears to be weaker in mouse cells than in human. Third, it requires DNA ends for activation. Exploring all of these facets of the SIDSP further will be of interest. Why is the response delayed? Does some transcriptional or cell biological event precede the IFN response? Now that we have provided the framework of the pathway and the sensor, studies elucidating the molecular mechanisms that govern the SIDSP will follow. Given the utility of mice in disease models, it will be important to more thoroughly determine whether the SIDSP is present in any mouse strain and/or what makes it less potent than it is in human cells. The lesson of making careful mouse-human comparisons has already been learned by the STING field - a potential cancer therapeutic DMXAA performed very well in mice but failed in human trials. Not coincidentally, DMXAA binds murine STING and activates a potent type I IFN response, but is completely unable to bid to human STING ${ }^{76}$. Surveying a variety of mouse strains and cell lines to thoroughly establish a mouse-specific deficiency in SIDSP signaling will be an important first step in clarifying this issue. One possible explanation could be a simple difference of expression, as there is evidence that DNA-PK is more highly expressed in humans than rodents ${ }^{77}$. Alternatively, there could be sequence-specific 
differences between human and murine DNA-PK or perhaps a unique human SIDSP co-factor that explains the lackluster response in mouse cells.

As outlined in the introduction, others have previously drawn connections between DNA-PK and IFN. However, this has always been in context of STING and either the requirement for the catalytic activity of DNA-PK was not required ${ }^{50}$ or not assessed ${ }^{52}$. In contrast, Nu7441 is a potent inhibitor of nearly all DNA-induced transcriptional activity in STING KO U937 cells. Additionally, Dr. Geoffrey Smith's body of work on DNA-PK and IFN was almost entirely conducted in mouse cells and/or mice, while we don't find compelling evidence of a strong SIDSP in these cells. Whether mouse deficient for both STING and DNA-PK completely lose the IFN response to DNA just as the human cells do is a key clarifying question.

Finally, in drawing a connection between the kinase activity of DNA-PK and IRF3 phosphorylation, we realize that there are obvious holes in the signaling pathway. If the SIDSP functions like the other known nucleic acid sensing pathways, what is the signaling adaptor, the STING analog? Would this analog also possess the $p$ LxIS motif? The identity of the direct IRF3 kinase is also unclear. TBK1 is not required for the SIDSP in HEK 293 cells, but DNA-PK prefers SQ/TQ motifs, which are not present in the $\mathrm{C}$ terminus of IRF3 ${ }^{78}$. This suggests that DNA-PK phosphorylates a non-canonical motif or that it activates an additional, as-of-yet unidentified kinase. Finally, it seems unlikely that cytosolic DNA activates a nuclear protein to activate a cytoplasmically-located transcription factor so that it can translocate to the nucleus. Likely the relevant pool of DNA-PK is the cytosolic fraction. Demonstrating whether this pool is activated by cellular fractionation after DNA transfection and assaying for markers of DNA-PK activation, such as autophosphorylation sites, could be a potentially illuminating way forward. 


\subsection{Figures}

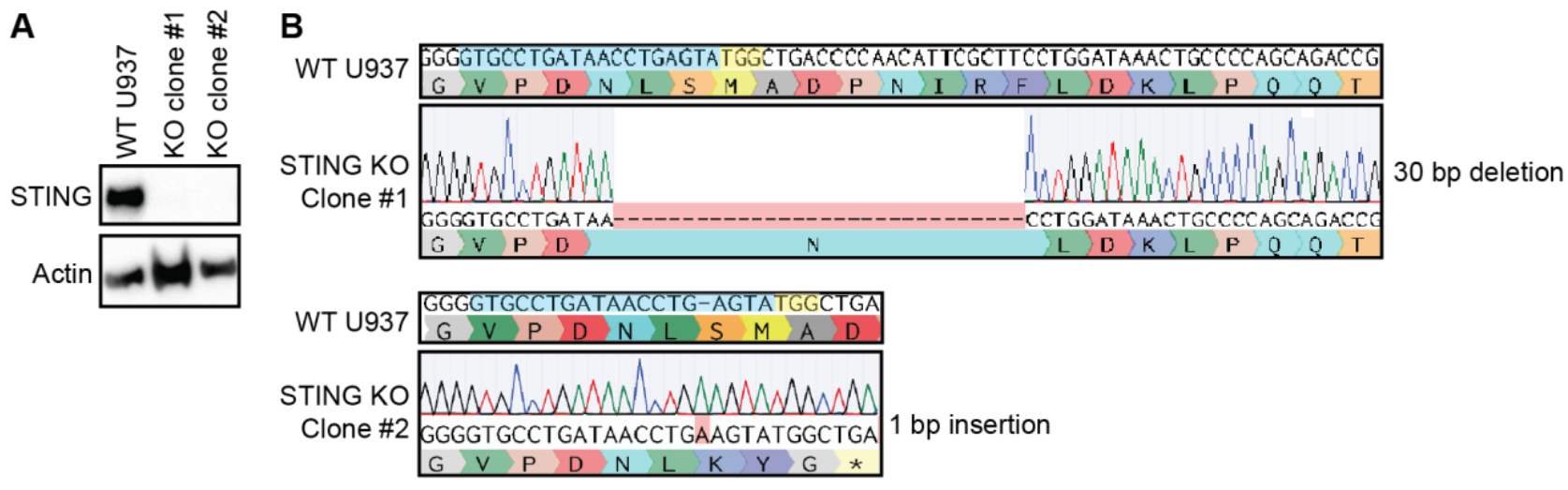

Figure 3: Generation of STING KO U937 clonal lines.

(A) U937 cells were transduced with lentiCRISPR encoding gRNAs specific for TMEM173 (STING), selected, cloned by limiting dilution, and then evaluated for STING protein expression by western blot.

(B) Clonal lines of HEK 293 cells were evaluated by Sanger sequencing, revealing a homozygous 30 bp deletion in Clone \#1 and a homozygous 1 bp deletion in Clone \#2. 

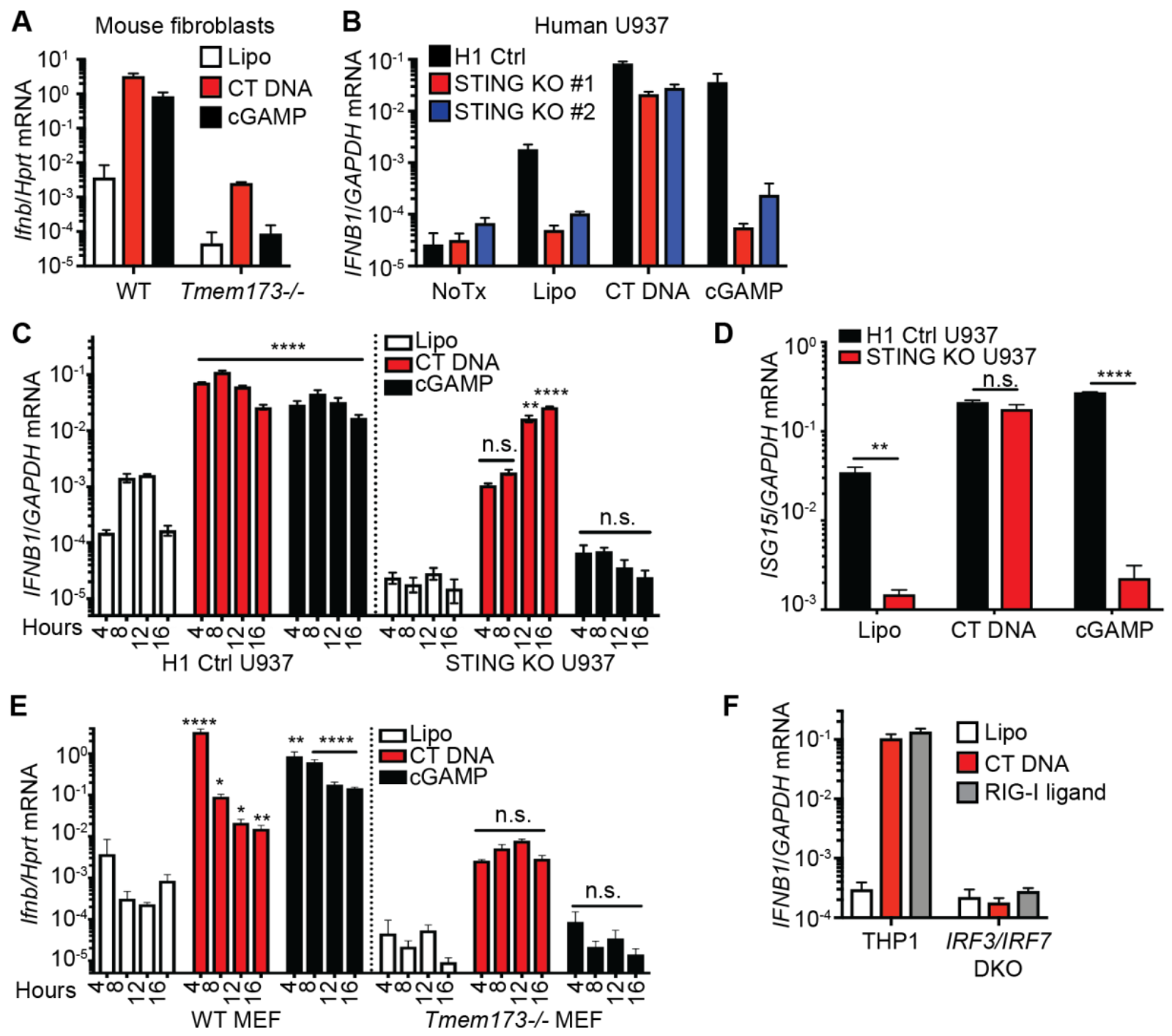

Figure 4: A STING-independent DNA sensing pathway in human cells.

(A) Primary mouse embryonic fibroblasts (MEFs) were treated with Lipofectamine alone (Lipo) or the indicated ligands for four hours before harvest and quantitative RT-PCR (qRT-PCR) analysis of Ifnb mRNA expression.

(B) PMA-differentiated H1 control and STING KO U937 cells were treated with the indicated ligands for 16 hours before harvest and qRT-PCR analysis of IFNB1 mRNA expression.

(C) PMA-differentiated $\mathrm{H} 1$ control U937 cells and STING KO U937 cells were treated with the indicated ligands for the indicated times before harvest and qRT-PCR analysis of IFNB1 mRNA expression. 2way ANOVAs were performed for each timepoint and Holm-Sidak's multiple comparisons test was used to compare CT-DNA and cGAMP treatments to lipofectamine alone. (D) PMA-differentiated WT U937 cells and STING KO U937 cells were treated with the indicated ligands for 16 hours before harvest and qRT-PCR analysis of ISG15 mRNA expression.

(E) WT and Tmem $173^{-\sim}$ MEFs were treated with the indicated ligands for the indicated times before harvest and qRT-PCR analysis of Ifnb mRNA expression. 2way ANOVAs were performed for each timepoint and Holm-Sidak's multiple comparisons test was used to compare CT-DNA and cGAMP treatments to lipofectamine alone. 
(F) PMA-differentiated THP1 cells and IRf3/IRF7 DO THP1 cells were treated with the indicated ligands for 16 hours before harvest and qRT-PCR analysis of IFNB1 mRNA expression. 

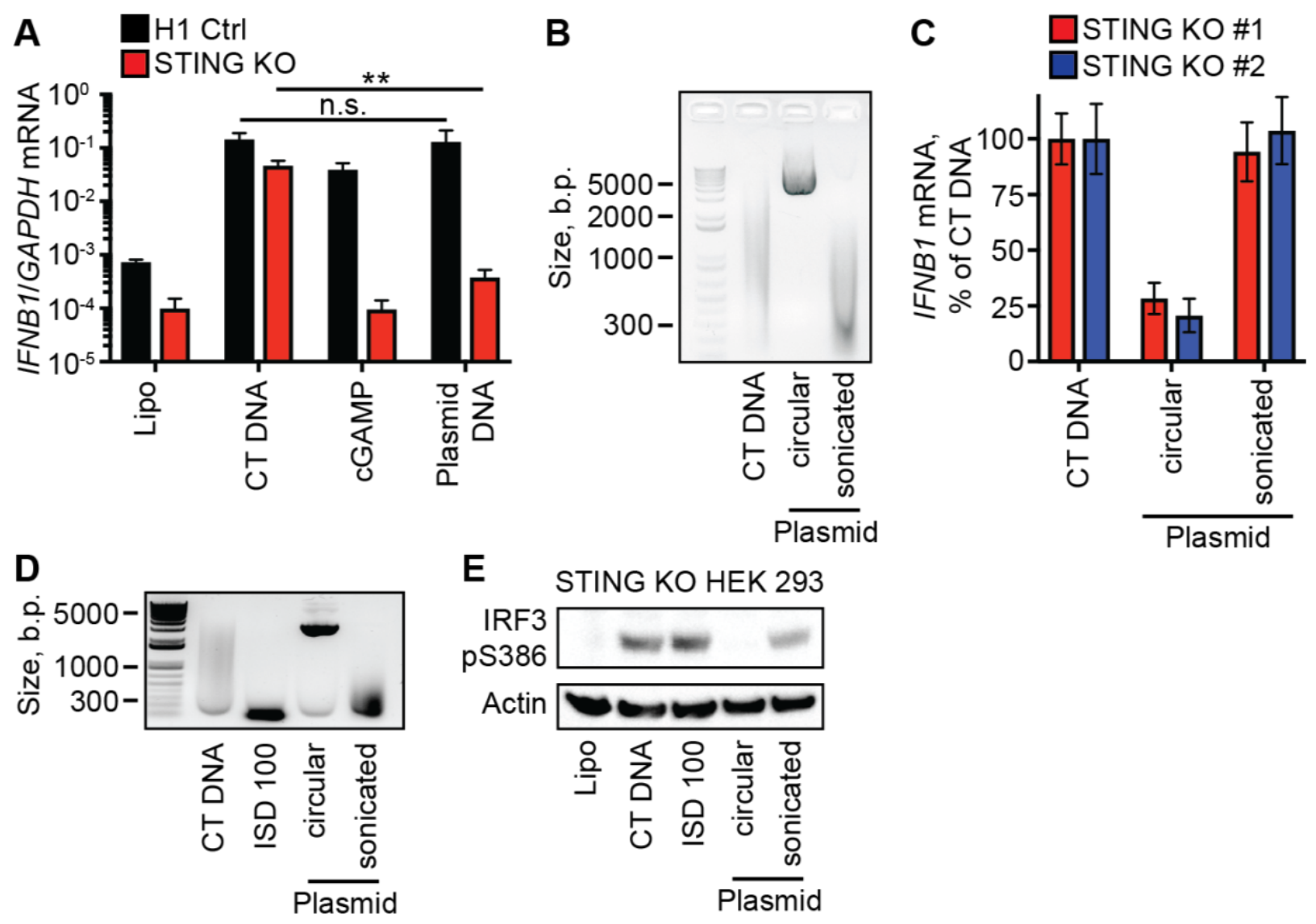

Figure 5: The SIDSP is activated by DNA ends.

(A) PMA-differentiated U937 cells and STING KO U937 cells were treated with the indicated ligands for 16 hours before harvest and qRT-PCR analysis of IFNB1 mRNA expression. n.s.: not significant; $* *: p<0.01$.

(B) CT DNA, supercoiled plasmid, and sonicated plasmid were run on a DNA agarose gel and visualized with SYBR-Safe.

(C) Two clonal lines of PMA-differentiated STING KO U937 cells were treated with the indicated ligands for 16 hours, with IFNBI mRNA expression in CT DNA-treated cells set at $100 \%$.

(D) CT DNA, 100 base pair annealed DNA oligos (ISD100), and either supercoiled or sonicated plasmid DNAs were visualized on a DNA agarose gel.

(E) STING KO HEK 293 cells were treated with the indicated ligands for three hours before harvesting lysates and evaluating IRF3 S386 phosphorylation by western blot. 

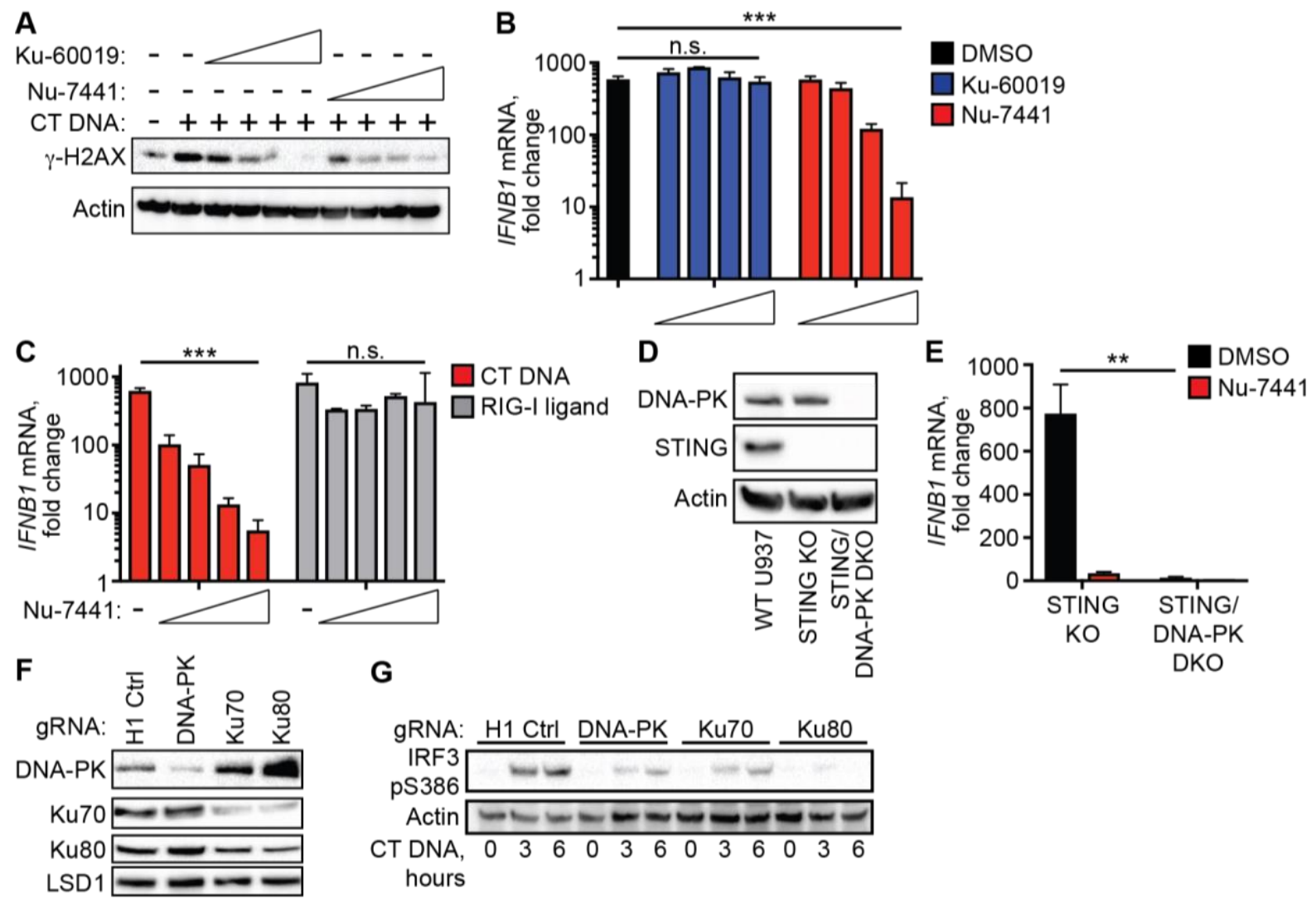

G

$$
\begin{aligned}
& \text { gRNA: } \mathrm{H}_{1 \mathrm{Ctrl}} \text { DNA-PK Ku70 Ku80 } \\
& \text { IRF3 }=- \\
& \text { Actin }-\infty-\infty-\infty-\infty-\infty-1 \\
& \text { CT DNA, } \begin{array}{llllllllllll}
0 & 3 & 6 & 0 & 3 & 6 & 0 & 3 & 6 & 0 & 3 & 6
\end{array}
\end{aligned}
$$

Figure 6: Human DNA-PK is essential for the SIDSP.

(A) PMA-differentiated STING KO U937 cells were treated with CT DNA for 16 hours in the presence of DMSO control or increasing concentrations of Ku-60019 ATM inhibitor (up to 1 $\mu \mathrm{M}$ ) or Nu-7441 DNA-PK inhibitor (up to $2 \mu \mathrm{M}$ ), followed by western blot analysis of $\gamma-\mathrm{H} 2 \mathrm{AX}$ phosphorylation.

(B) PMA-differentiated STING KO U937 cells were treated with CT DNA for 16 hours in the presence of inhibitors as described in (A), followed by qRT-PCR analysis of IFNB1 mRNA expression. n.s.: not significant; $* * *: p<0.001$.

(C) PMA-differentiated STING KO U937 cells were treated with CT DNA or RIG-I ligand in the presence of DMSO or Nu-7441 for 16 hours, followed by qRT-PCR analysis of IFNBI mRNA expression. n.s.: not significant; ***:p<0.001.

(D) Western blot analysis of DNA-PK and STING in WT U937 cells and clonal lines of STING KO and STING/DNA-PK DKO U937 cells.

(E) PMA-differentiated STING KO and STING/DNA-PK DKO U937 cells were treated with CT DNA for 16 hours in DMSO or $2 \mu \mathrm{M}$ Nu-7441, followed by qRT-PCR analysis of IFNB1 mRNA expression, normalized to Lipo control-treated cells. $* *: p<0.01$.

(F) STING KO HEK 293 cells were transduced with lentiCRISPR encoding gRNAs specific for the indicated targets, selected for three days in puromycin, and then harvested for western blot analysis of the indicated proteins.

(G) STING KO HEK 293 cells from (F) were stimulated with CT DNA for the indicated time points and then harvested for western blot analysis of IRF3 S386 phosphorylation. 

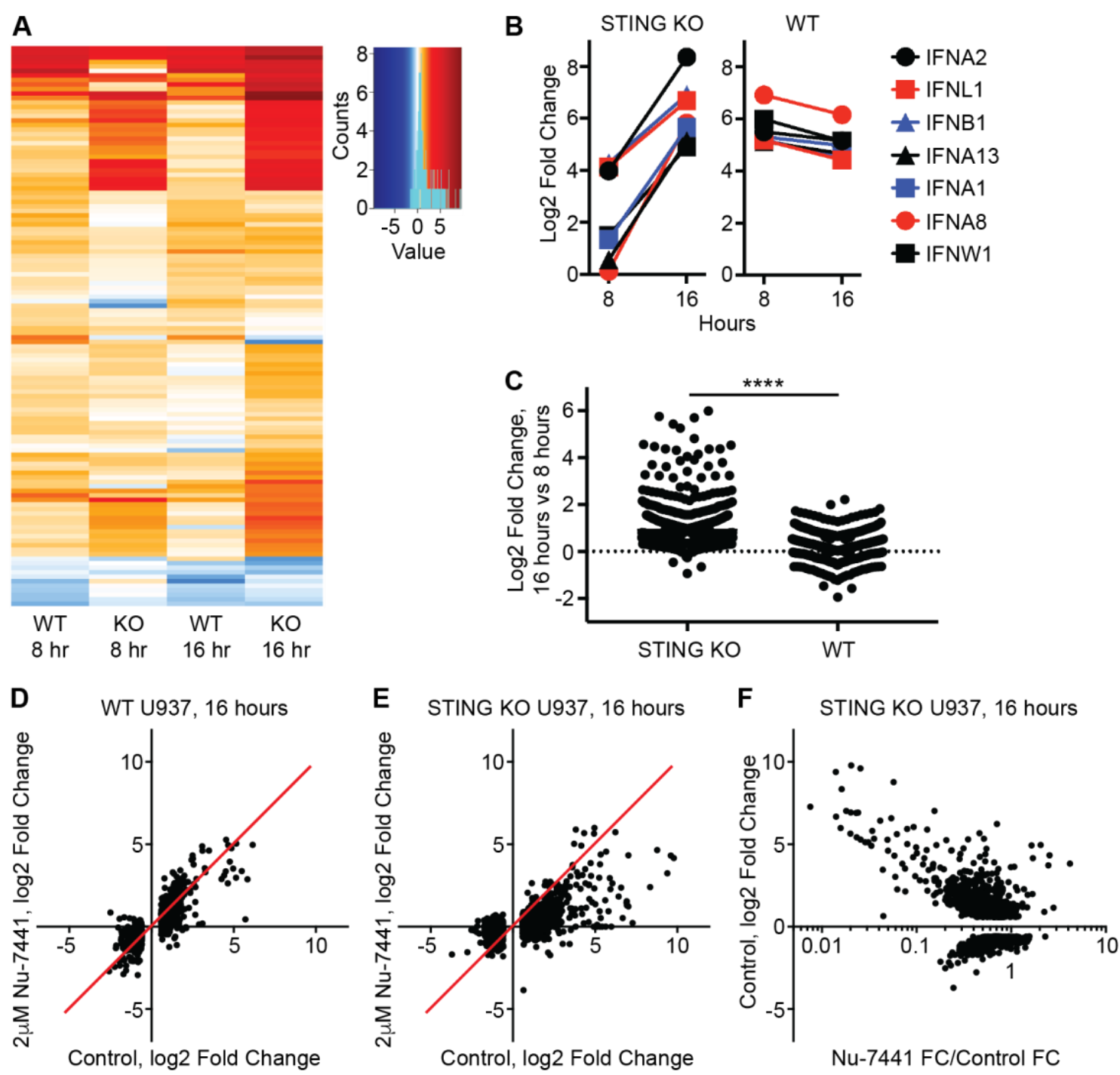

Figure 7: The DNA-PK SIDSP activates a broad gene expression program.

(A) Heat map representation of $\log 2$ Fold Change in gene expression for the 124 antiviral response genes with significant differential expression in one of the four comparisons. The key includes a histogram in cyan plotting the distribution of $\log 2$ Fold Change values for all the included genes.

(B) Expression data for all interferon genes significantly induced in WT or STING KO U937 cells, plotting the $\log 2$ Fold Change at 8 and 16 hours post treatment.

(C) To measure the trajectories of global gene expression from 8 to 16 hours post CT DNA transfection, the fold change in gene expression at 16 hours was divided by the fold change for the same genes at 8 hours and plotted for all upregulated genes in STING KO $(n=926)$ and WT $(\mathrm{n}=563) . * * * *: \mathrm{p}<0.0001$.

(D) In WT U937 cells, the 16 hour CT DNA-activated log2 Fold Change was plotted for DMSO control-treated cells on the $\mathrm{x}$-axis and for $\mathrm{Nu}$-7441-treated cells on the $\mathrm{y}$-axis. Each dot represents a single gene that was differentially expressed at 16 hours in DMSO-treated cells $(n=1024)$. The red line through the origin indicates the line of equivalence representing no effect of the drug treatment. 
(E) In STING KO U937 cells, the effect of Nu-7441 on global gene expression for 1327 genes differentially expressed in DMSO control-treated cells, plotted as in (E).

(F) In STING KO U937 cells, the log2 Fold Change in DMSO control-treated cells was plotted on the $\mathrm{y}$-axis for differentially expressed genes, and the effect of $\mathrm{Nu}-7441$ on these same genes was plotted in $\log 10$ format on the $y$-axis, $n=1327$. 


\section{Chapter 4: Identification of a specific marker of the SIDSP}

\subsection{Introduction}

During experiments involving western blotting for pIRF3 S386, we routinely observed a DNA-induced band that was roughly $70 \mathrm{kDa}$ (Fig. 8A), that we aptly referred to as Mystery Protein (MP). This band was present not only in HEK 293s, but in all human cell lines tested including primary fibroblasts (Fig. 8B-D). This "mystery protein" was not induced by RIG-I ligand, suggesting that it may be specific to the SIDSP. Treatment of lysates with phosphatase reduced the signal (not shown), suggesting that MP was a phosphoprotein. Here we identify this protein as phosphorylated HSPA8 using immunoprecipitation and mass spectrometry and characterize its relationship to the SIDSP.

\subsection{Results}

\section{Identification of a DNA-induced phosphoprotein}

To test whether STING or TBK1 were required for Mystery Protein (MP) induction, we used the respective KO HEK 293 lines previously generated and found that both CT DNA and ISD100 induced MP in both cell lines (Fig. 8E). Additionally, we found that MP was activated by sonicated plasmid, but not circular plasmid (Fig. 8F), suggesting that MP phosphorylation correlates with the SIDSP and could be a readout for SIDSP activation. To identify MP, we used the pIRF3 S386 antibody to immunoprecipitate from CT DNA-stimulated HEK 293 cells, digested the proteins with trypsin, and analyzed the samples by mass spectrometry. One of the enriched peptides was from heat shock protein A8 (HSPA8, also called HSC70), a $73 \mathrm{kDa}$ chaperone protein. Strikingly, HSPA8 possesses a sequence "GGASS" in its C terminus that matches the surrounding residues of IRF3 S386 (Fig 8G), which could explain why it was detected using the antibody raised against pIRF3 S386. HSPA8 S638 corresponds to IRF3 S386, 
but IRF3 can be phosphorylated on S385 as well, so in order to determine whether MP was, in fact, HSPA8, we cloned and epitope-tagged human HSPA8 from HEK 293 cells, generating serine to alanine mutant versions at positions 637,638 , or both. After transient transfection of the constructs into HEK 293 cells and subsequent DNA stimulation, we immunoprecipitated with an anti-HA antibody and blotted for pIRF3/MP. Importantly, the WT, serine-containing construct, and not the S637A or S638A alanine mutants, was detected by the pIRF3 S386 antibody, indicating that the MP recognized by the antibody is HSPA8 (Fig. 8H).

\section{Phosphorylation of HSPA8 is conserved across species}

HSPA8 is a member of the Hsp70 family of heat shock chaperone proteins that is highly conserved across primates and rodents (Fig. 9A). Therefore, we hypothesized that the SIDSP might exist in other species besides human, and that we might be able to screen for the SIDSP using the same pHSPA8 S638 antibody as previously utilized. To screen for the SIDSP across species, we blotted lysates from a panel of primate and rodent cell lines with the pHSPA8specific antibody. Interestingly, we observed potent DNA-induced pHSPA8 in all lines tested, excepting mouse cells (Fig. 9B). This ubiquitous DNA-induced HSPA8 phosphorylation across primate and rodent cell lines demonstrates that the SIDSP is present across species, and also corroborates our data showing that the SIDSP is weak in mouse cells. To determine if this mouse-specific deficiency is due to the single amino acid difference between mouse and human HSPA8 (amino acid 578), we cloned WT and S637/638 alanine mutant murine HSPA8 and performed reciprocal experiments, expressing tagged human and mouse versions of HSPA8 in both human and mouse cells. We found that mouse HSPA8 was phosphorylated in human cells and that neither human, nor mouse HSPA8 was phosphorylated in mouse cells (Fig. 9C-D). This result suggests that the lack of DNA-induced pHSPA8 is not due to differences between human 
and murine HSPA8 but instead some defect in the SIDSP machinery in mouse cells. Exploring how and why exactly mice appear to have lost the SIDSP will be of interest. It appears that rat cells are competent for the SIDSP (Fig. 9B), suggesting that mouse-rat comparisons could be informative.

Phosphorylated HSPA8 is a marker for the SIDSP

We turned again to lentiCRISPR to address the role of DNA-PK, Ku70, and Ku80 in HSPA8 phosphorylation in HEK 293 cells and found that all three are required (Fig. 10A); additionally, we observed that targeting ATM did not reduce the DNA-induced pHSPA8 or pIRF3 (Fig. 10BC). These data are consistent with our results from U937s, showing that the ATM inhibitor did not block the SIDSP (Fig. 6B). We were intrigued to see that targeting DNA-PK resulted in a loss of ATM expression, but this is consistent with previous literature ${ }^{79}$. Finally, we tested the effect of HSV-1 ICP0, a ubiquitin ligase known to degrade DNA-PK ${ }^{80}$, on the SIDSP in HEK 293 cells and we saw a dose-dependent decrease in both pHSPA8 and pIRF3 (Fig. 10D). These data demonstrate that pHSPA8 tracks with the SIDSP in every discernible way, suggesting that it could be used as a convenient marker for the SIDSP. Additionally, we have identified ICP0 as a second virally-encoded inhibitor of the SIDSP, suggesting that multiple viruses have encoded proteins to inhibit the SIDSP in host cells.

The antiviral modality of DNA-PK is distinct from its DNA damage modality

Finally, we sought to explore whether activation of DNA-PK in general also activates the SIDSP. As DNA-PK is known to be activated in response to DNA damage, we utilized both ionizing radiation and etoposide to damage DNA and assayed for $\gamma$-H2AX and pIRF3/pHSPA8 as readouts for DNA damage and the SIDSP, respectively. We found that CT DNA transfection induces potent $\mathrm{pHSPA} 8$, pIRF3 and $\gamma-\mathrm{H} 2 \mathrm{AX}$, in contrast to circular plasmid DNA, which 
activates none of these (Fig. 11). However, neither ionizing radiation nor etoposide induced pHSPA8/pIRF3, despite activating strong $\gamma$-H2AX responses. These data indicate that there are different activation "modalities" of DNA-PK - the canonical DNA damage modality, which does not result in pIRF3 or pHSPA8, but activates $\gamma-\mathrm{H} 2 \mathrm{AX}$; and an antiviral modality, which activates both pHSPA8 and pIRF3.

\subsection{Discussion}

Here we discover that HSPA8 is phosphorylated in response to transfected sheared DNA, suggesting that, in addition to its canonical role as a chaperone that mediates protein folding and degradation, it may also play a role in the SIDSP. Also, this is the first report of inducible, sitespecific host modification of HSPA8. Interestingly, a pathogen-mediated modification of HSPA8 has just recently been reported: Legionella was shown to encode a kinase that phosphorylates HSPA8 (and other Hsp70s) at T495 to slow protein synthesis in host cells ${ }^{81}$. The functional significance of phosphorylation at S638 is unknown, but adjacent to that site is an EEVD motif. This motif is found in other heat shock proteins and allows for binding of tetratricopeptide repeat (TPR) containing co-chaperones. These co-chaperones determine the client proteins targeted by the heat shock proteins and as well as their fates - for example, whether they will be degraded or folded. In vitro studies show that replacing the serines in the C terminus of Hsp90 and Hsp70 with phosphomimetic residues affects binding by co-chaperones. Thus, we hypothesize that DNA-induced phosphorylation of HSPA8 will affect its affinity for one or more co-chaperones, which would affect its targets and function. It is interesting that HSPA8 is required for STING degradation by autophagy ${ }^{31}$, but whether phosphorylation of HSPA8 affects this process is currently unknown. 
We observe that the phosphorylation of HSPA8 correlates with the SIDSP - it is not activated by RIG- I ligand, cGAMP, or circular DNA; it is independent of STING and TBK1; it requires DNA-PK, Ku70 and Ku80; it is wide-spread across human (and primate) cell lines, but not induced in mouse cells. Interestingly, fact that HSPA8 is so conserved makes pHSPA8 a convenient marker for the SIDSP across species and will be a useful readout for future work. Further probing the role of HSPA8 in the SIDSP will be important. Is it a positive or negative regulator, or does it simply correlate with the SIDSP? Is phosphorylation required for function? KO studies are impossible due to HSPA8's role in homeostasis, so overexpression and inhibitor studies will be necessary to begin to answer these questions.

Finally, we observe context-specific responses of DNA-PK to broken DNA ends. After endogenous DNA damage, broken ends are recognized by DNA-PK, but do not induce an antiviral response. In context of transfected linear DNA, ends are bound by DNA-PK and perceived as so great a threat as to provoke a strong antiviral response. What prevents a DNAPK mediated antiviral response to DNA damage? This is a major question for the future. 


\subsection{Figures}

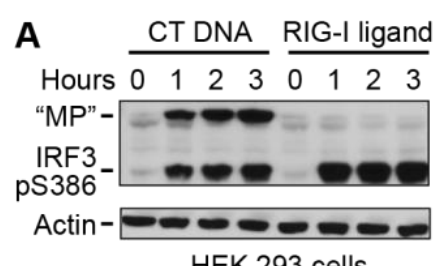

HEK 293 cells

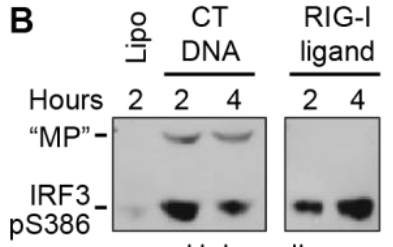

HeLa cells

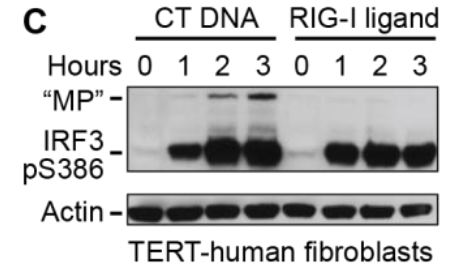

TERT-human fibroblasts
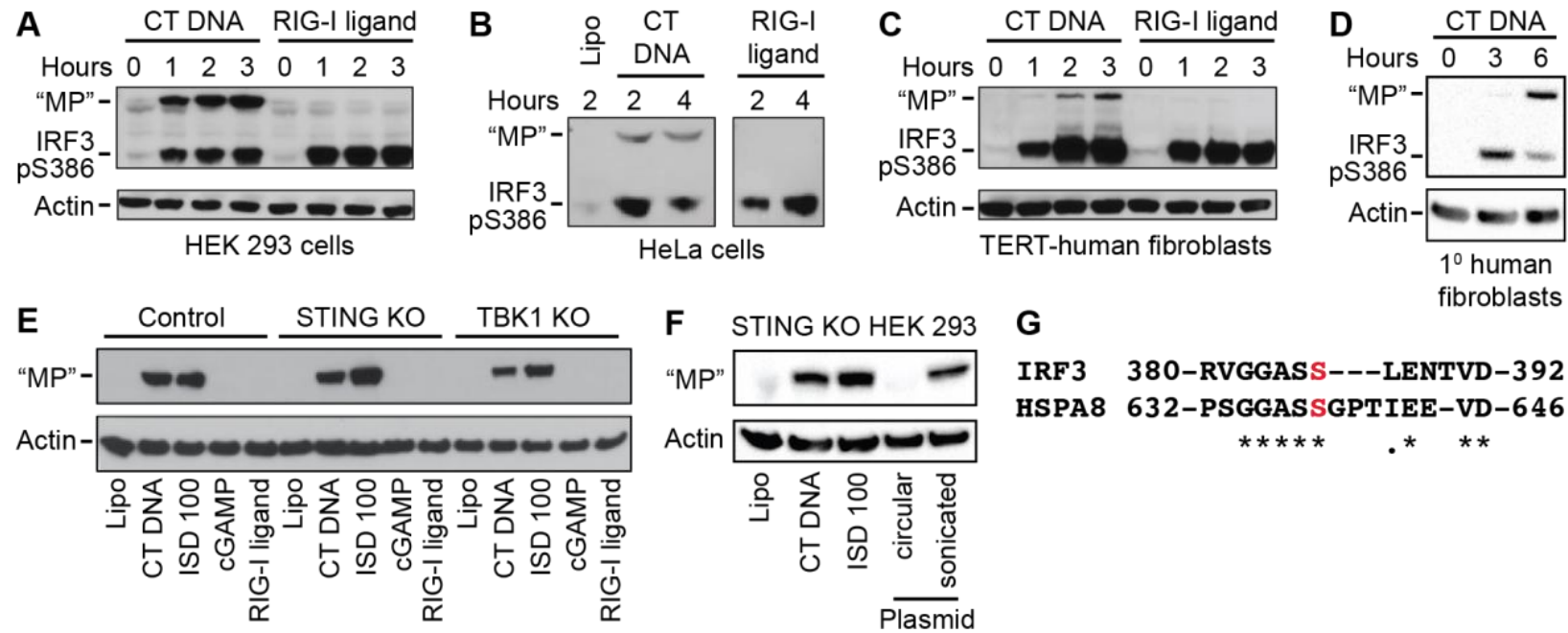

F STING KO HEK 293

G

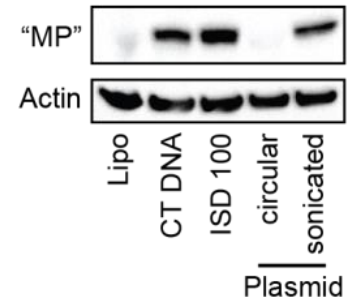

IRF3 380-RVGGASS---LENTVD-392

HSPA8 632-PSGGASSGPTIEE-VD-646
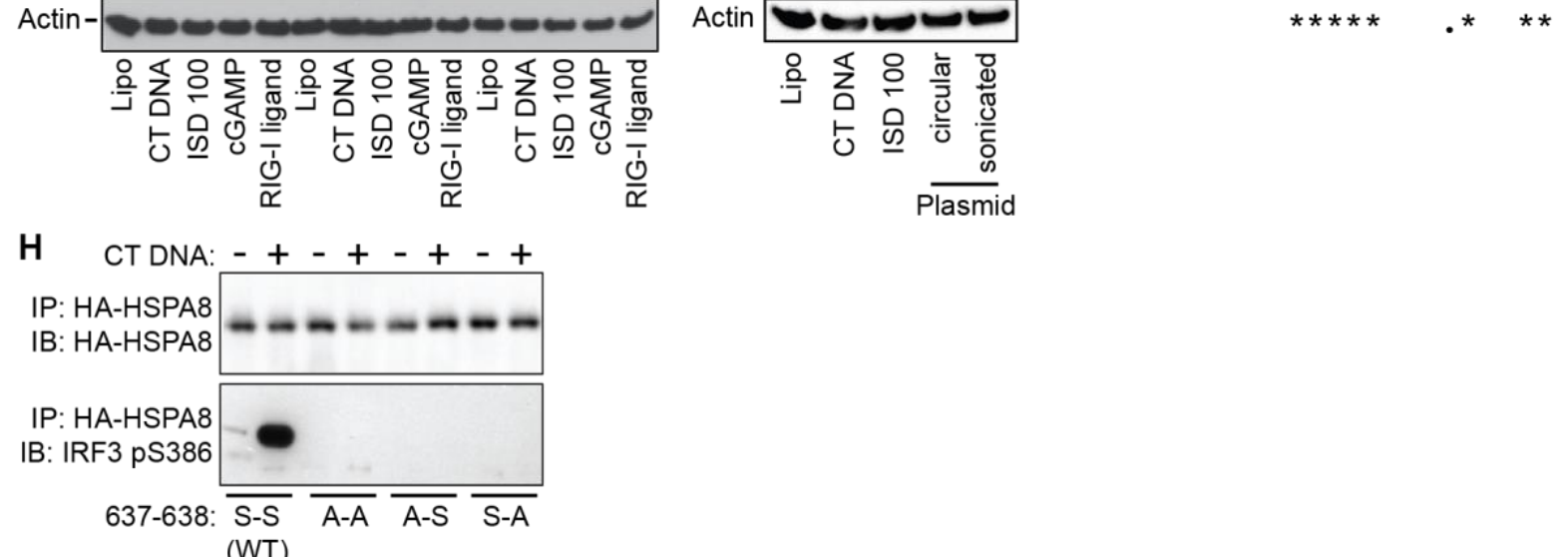

(WT)

Figure 8: HSPA8 is a target of the DNA-PK mediated SIDSP.

(A-D): The indicated human cells were treated with CT DNA or RIG-I ligand for the indicated tims before harvest and western blot analysis of IRF3 S386 phosphorylation. "Mystery Protein" ("MP") is indicated on the blots.

(E) Control, STING KO, and TBK1 KO HEK 293 cells were treated with the indicated ligands for 3 hours before harvest and western blot analysis of "MP."

(F) STING KO HEK 293 cells were treated with the DNA ligands for 3 hours, followed by western blot analysis of "MP."

(G) Alignments of human IRF3 and HSPA8/HSC70. The red S indicates IRF3 S386 and HSPA8 S638.

(H) HEK 293 cells were transfected with plasmids encoding the indicated human HA-HSPA8 constructs, then treated the next day with CT DNA for 3 hours before harvest, HAimmunoprecipitation, and western blot using the IRF3 pS386 antibody. 
A

\begin{tabular}{r|l} 
Hominoids & $\begin{array}{l}\text { Homo sapiens } \\
\text { Pan troglodytes }\end{array}$ \\
Old World & $\begin{array}{l}\text { Macaca mulatta } \\
\text { Monkeys }\end{array}$ \\
New World & $\begin{array}{l}\text { Aotus trivirgatus } \\
\text { Monkeys } \\
\text { Saimiri boliviensis }\end{array}$ \\
Rodents & $\begin{array}{l}\text { Mesocricetus auratus } \\
\text { Rattus norvegicus } \\
\text { Mus musculus }\end{array}$
\end{tabular}

HSPA8

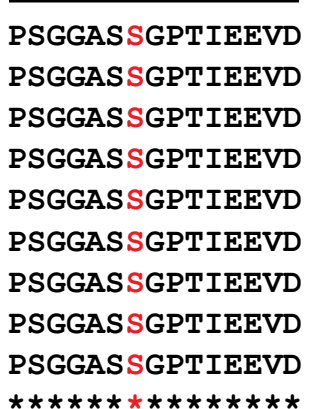

IRF3

MARVGGASSLENTVDL MARVGGAS SLENTVDL IARVGGAS SLENTVDL IARVGGAS SLENTVDL LARVGGAS SLENTVEL LARVGGAS SLENTVEL MAREGGASSLR-TVDL MAREGGASSLK-TVDL MAREGGASSLK-TVDL

B

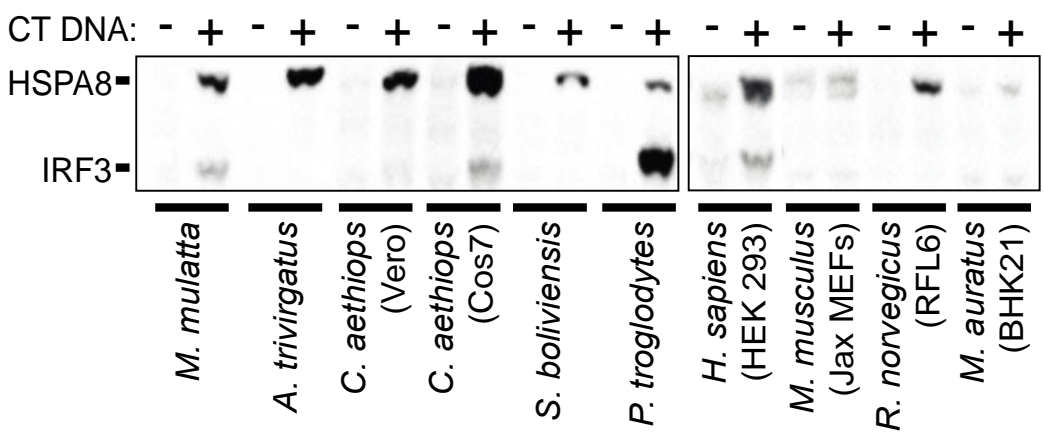

C

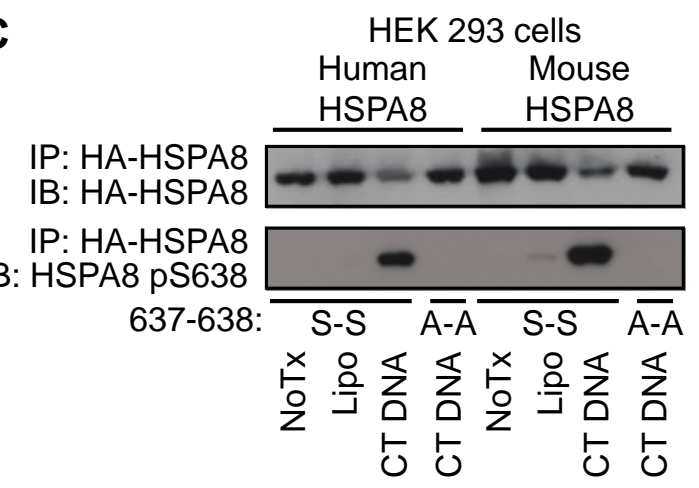

D

D Mouse fibroblasts

Human Mouse

HSPA8 HSPA8

IP: HA-HSPA8

IB: HA-HSPA8

IP: HA-HSPA8

IB: HSPA8 pS638

637-638: $\overline{S-S} \overline{A-A} \overline{S-S} \overline{A-A}$

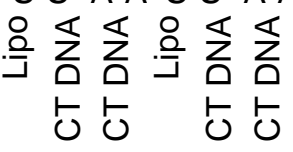

Figure 9: Phosphorylation of HSPA8 is conserved across species.

(A) Alignments of HSPA8 and IRF3 amino acid sequences surrounding the phosphorylated serines.

(B) The indicated human, primate, and mouse cell lines were stimulated with CT-DNA for 3 hours before harvest and western blot for the indicated proteins.

(C) HEK 293 cells were transfected with either human HA-HSPA8 constructs or mouse HAHSPA8 constructs, followed by CT DNA stimulation for 3 hours, HA immunoprecipitation, and western blot analysis of the indicated proteins.

(D) Mouse immortalized Jackson fibroblasts were transfected and treated as indicated in (C). 


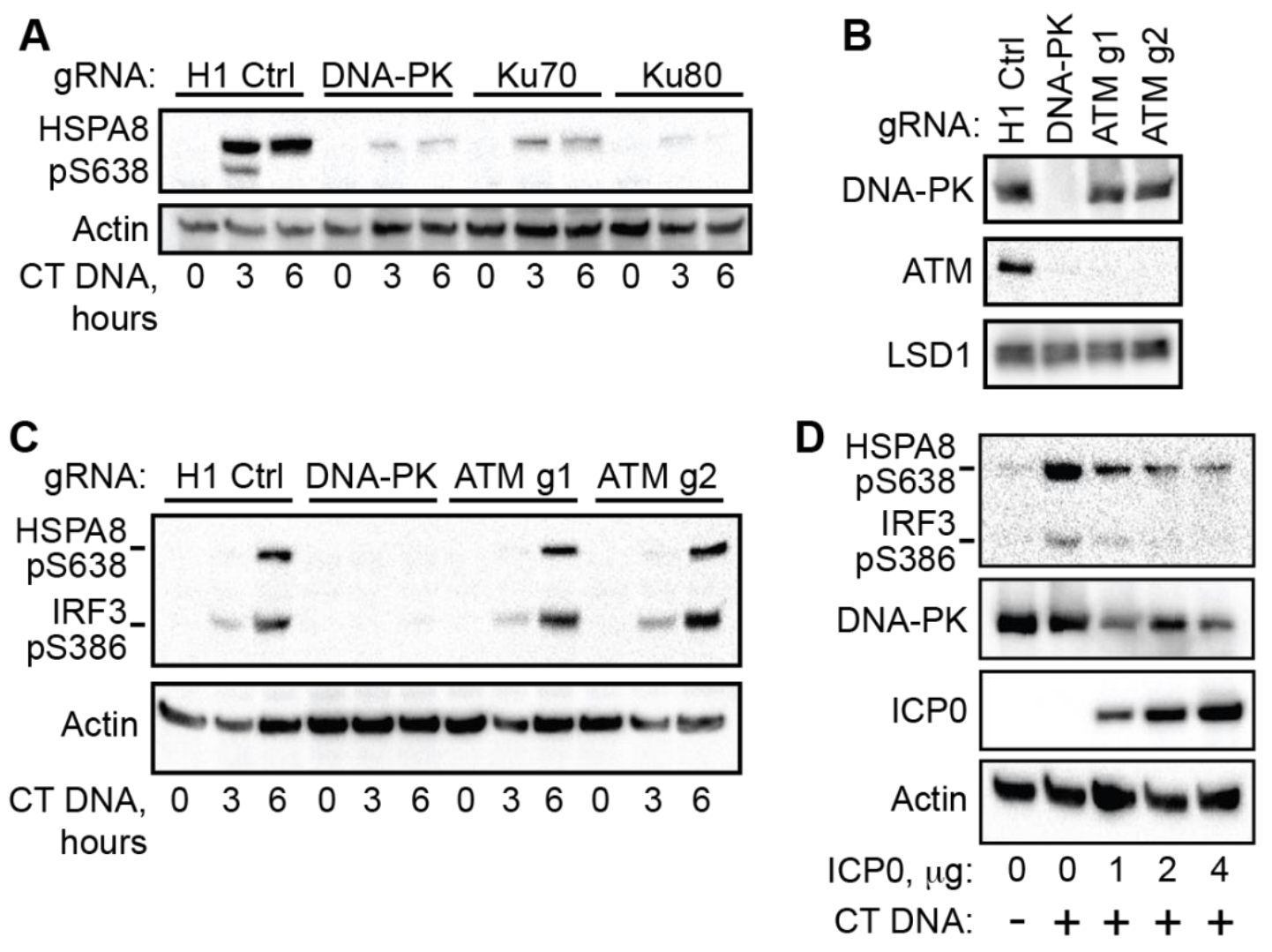

Figure 10: Phosphorylation of HSPA8 is a marker for the SIDSP.

(A) HEK 293 cells targeted for the indicated genes were treated with DNA and harvested for western blot analysis using the IRF3 pS386 antibody that detects HSPA8 pS638.

(B) STING KO HEK 293 cells were transduced with lentiCRISPR targeting H1 control, DNAPK, or ATM, selected for three days, and then harvested for western blot of the indicated proteins.

(C) STING KO HEK 293 cells transduced and selected and then treated with CT-DNA and then harvested for western blot analysis of IRF3 S386 and HSPA8 S638 phosphorylation.

(D) STING KO HEK 293 cells were transfected with plasmid encoding the ICP0 protein of herpes simplex virus 1. 24 hours later, the cells were stimulated with CT DNA for 3 hours before harvest and western blot analysis of the indicated proteins. 


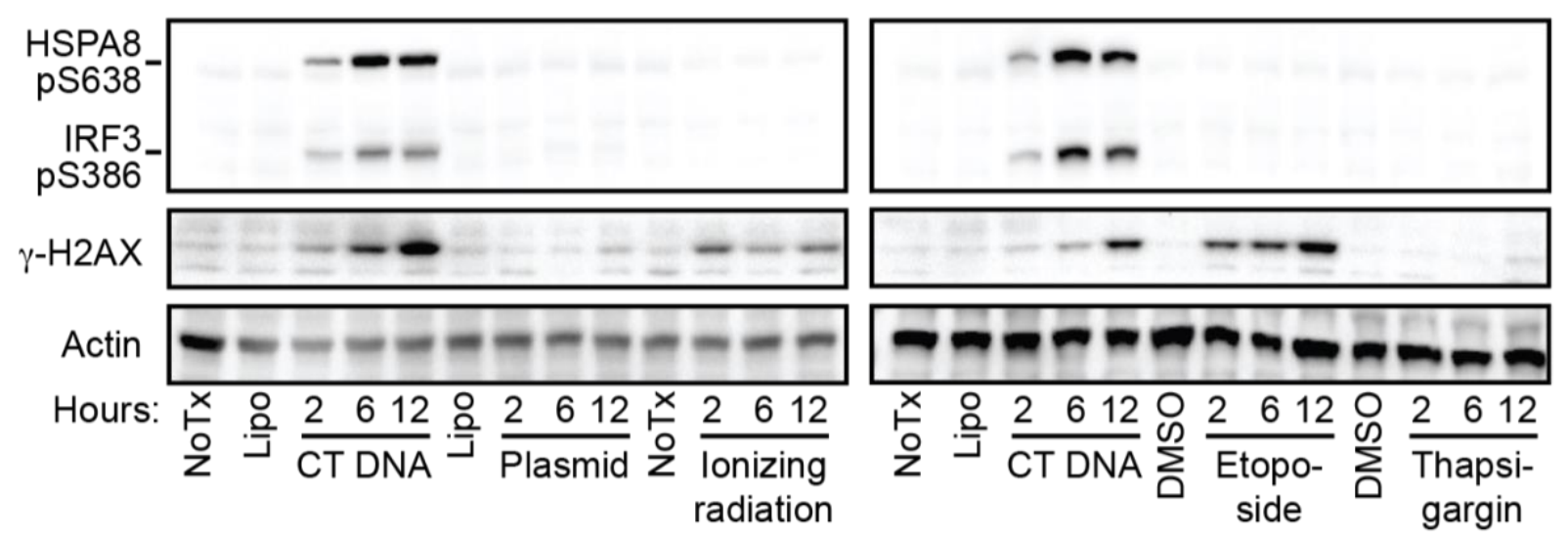

Figure 11: pHSPA8 delineates an antiviral modality for DNA-PK.

HEK 293 cells were stimulated with CT DNA or supercoiled plasmid DNA, or treated with 30 Gray ionizing irradiation, 50mM Etoposide, or $500 \mathrm{nM}$ Thapsigargin before harvest at the indicated time points and western blot analysis of IRF3 pS386, HSPA8 pS638, and $\gamma$-H2AX. 


\section{Chapter 5: Conclusions}

\subsection{Introduction}

The existence of what would become known as the cGAS-STING pathway was discovered in $2006^{10}$. The presence of an IRF3-dependent, TLR9-independent antiviral response to transfected DNA was not immediately regarded by all as important; some termed this pathway "the transfection response" as if it were a mere artifact. In 2008 and 2009, two studies linked STING to DNA-induced antiviral immunity ${ }^{11,13}$. Bacterial cyclic dinucleotides were shown to bind and activate murine STING, but the sensor required for DNA was unknown for many years, until Dr. Zhijian Chen's group published the discovery of both cGAS and an endogenous dinucleotide, cGAMP, in 2012 21,22 . The first DNA virus encoded antagonists of the cGAS-STING pathway were not published until 2015, nearly a decade after the discovery of the pathway itself ${ }^{58,61-63}$. Here we report the existence of a parallel STING-independent DNA sensing pathway (SIDSP). We identify the sensor, a specific marker, and two viral antagonists of the pathway.

\subsection{Potential relevance of the SIDSP in context of cancer and autoimmunity}

Just as the cGAS-STING pathway has been shown to be important in context of cancer and autoimmunity, so we predict that the SIDSP will also be widely relevant across biomedical fields. One of the major hurdles in cancer therapy is the typically immune suppressive environment of the tumor. In mouse models, intratumoral injection of STING agonists has been shown to reduce tumor growth at both injected and distal sites through activation of macrophages and dendritic cells in the tumor microenvironment ${ }^{82,83}$. However, likely in order to escape the immune system, some tumors downregulate STING expression ${ }^{84}$, which would make this strategy potentially less efficacious. Perhaps in patients with tumors that express low levels of STING, a SIDSP agonist would be a more effective treatment. 
Regulation of nucleic acid sensing is critical to prevent autoimmune disease. Loss of negative regulation and gain-of-function mutations have both been shown to cause dysregulation of cGAS-STING signaling. Loss-of-function mutations in the DNA exonuclease TREX1 gene cause Aicardi-Goutières Syndrome (AGS), a disease that presents in infancy with a severe phenotype similar to an in-utero viral infection. Trex $1^{-/}$mice develop severe autoimmune pathology and have compromised survival that is rescued by crossing to mice deficient in cGAS or IRF $3^{85}$, demonstrating that TREX1 plays a role in degrading endogenous DNA molecules, preventing them from aberrantly activating the cGAS-STING axis and causing unneeded inflammation. Conversely, gain-of-function mutations in STING have been shown to cause both familial chilblain lupus, as well as an inflammatory disease termed STING-associated vasculopathy with onset in infancy (SAVI) ${ }^{86,87}$. The discovery of mutations in regulatory factors or main components of the SIDSP that lead to aberrant IFN and autoimmune disease will demonstrate the biological relevance of the SIDSP and provide valuable models to study the pathway and its regulation.

\subsection{Potential relevance of the SIDSP in context of DNA virus infection}

The fact that two viruses, adenovirus and HSV-1, encode antagonists of the SIDSP is evidence of its importance. Many questions remain - what are the viral SIDSP ligands? When and where is this DNA recognized? What other viral factors restrain the SIDSP to promote replication? There is ample evidence of viral hijacking of DDR; some viruses require DDR to replicate properly, and others efficiently antagonize the NHEJ or the HR pathways ${ }^{88}$. It will be informative to explore whether the function of some DNA-PK antagonists is to block the SIDSP. Given our data on E1A and ICP0, we are particularly interested in exploring how adenovirus and HSV-1 interface with the SIDSP. 
Adenovirus has a linear dsDNA genome and thus replication requires modulation of the DDR. E4-deficient adenovirus becomes linked into multi-genome concatemers that are too large for packaging, indicating that the cell does recognize the genome as a substrate for 'repair' 89 . Cells deficient for either NHEJ factors (DNA-PK or Ligase 4) or HR factors (Mre11 or NBS1) rescue concatermerization of the mutant. Two of the E4 open reading frames, E4orf3 and E4orf6, are redundantly required for degradation of the members of the MRN complex (Mre11, Rad50, NBS1 $)^{89}$ and also the binding and inactivating of DNA-PK ${ }^{90}$.

A recent study suggests that another adenovirus protein, E1A, may impact the DDR by binding $\mathrm{Ku} 70^{91}$. E1A is the first translated adenoviral protein and an essential Swiss army knife that both promotes viral transcription and modulates host biology, most notably by inducing Sphase in quiescent cells. It has been described as an "invasive hub protein" - it is a flexible, disordered protein with many short linear interaction motifs (SLiMs) that allow it to make contact with over 32 primary targets, and 2,000 secondary targets ${ }^{92}$. Here we demonstrate that E1A blocks both SIDSP and STING signaling, which highlights the efficiency of E1A antagonism of innate immunity. This is further highlighted by the fact that it not only antagonizes the induction of IFN, but also signaling downstream of IFN by blocking a key histone modification required for transcription of $\mathrm{ISGs}^{93}$.

It will not be straightforward to demonstrate that 1) the SIDSP is active during viral infection and 2) blocking the SIDSP is required for infection. First, because viruses encode antagonists of the SIDSP, we may be unable to measure pHSPA8 or IFN during WT infection. Second, experiments testing whether prevention of adenoviral antagonism of the SIDSP reduces replication may be difficult to interpret. E1 A-null viruses are already unable to replicate due to E1A's role in promoting viral transcription, and although many E1 A mutant viruses exist, given 
the sheer number of E1A binding partners, it is likely that a mutant deficient in SIDSP antagonism would also be deficient for some other E1A function. A role for the SIDSP in the phenotype of a potentially poorly replicating mutant could be established by rescue with $\mathrm{Nu} 7441$ (DNA-PK inhibitor). However, even a highly specific loss-of-function E1A mutant may not result in a virus that does not block the SIDSP. Just as KSHV antagonizes DNA sensing with three unique antagonists and vaccinia blocks DNA-PK with two, it is likely that adenovirus encodes more than one antagonist of the pathway. E4orf3/orf6 are potential candidates given that they already block the DNA repair function of DNA-PK. Demonstrating the converse - that knockdown/inhibition of DNA-PK enhances viral replication because of relief from the SIDSP will be difficult because of the importance of DNA repair for cellular growth. A DNA-PK null human cell line exhibited extremely slow growth and a Ku80 heterozygous null line also had a growth defect ${ }^{75,73}$. Given the fact that adenovirus promotes its replication by inducing S-phase (and thus production of dNTPs), it is not surprising that $\mathrm{Ku} 70$ knockdown appears to limit viral titer ${ }^{94}$. Any advantage gained by loss of the SIDSP may be overwhelmed by the disadvantage of an inability to promote cell entry into S-phase. Given these complexities, it may be best to try simpler experiments to help understand viral interactions with the SIDSP. Viral proteins could be overexpressed in STING KO cells before DNA stimulation and measurement of IFN to determine whether there are additional antagonists of the pathway. Additionally, identifying a mutation or factor that somehow decouples the SIDSP from DNA repair/cellular growth would conceptually simplify these experiments.

The interaction of HSV-1 with DDR machinery is complex. Ligase 4 and XRCC4, important for the ligation step of NHEJ, are required for viral replication, while DNA-PK appears to be inimical for HSV-1 ${ }^{95,96}$. Perhaps this seeming contradiction indicates that non-NHEJ functions of 
DNA-PK are hostile to the virus. HSV-1 is a linear dsDNA virus, but fascinatingly, is riddled with nicks and gaps that could potentially be DNA-PK substrates ${ }^{96}$. However, HSV-1 encodes ICP0, a ubiquitin ligase that promotes degradation of DNA-PK in a proteasome dependent manner $^{80}$, and consistent with an important role in blocking host defenses, it is both expressed early in infection, and also found in the viral tegument ${ }^{96}$. Indeed transfection of purified viral genomic DNA potently activates DNA-PK, but infection of WT HSV-1 does not ${ }^{96}$. This indicates that the unique structure of the HSV-1 genome is recognized by DNA-PK, but during infection, ICP0 prevents DNA-PK activation. Interestingly, the ICP0-null mutant, but not WT HSV-1, induces IRF3-dependent ISG expression in infected cells ${ }^{98}$. Testing whether the DNAPK and IRF3 antagonizing functions of ICP0 are linked via the SIDSP is of interest.

\subsection{Conclusion}

Here we have presented a novel nucleic acid sensing pathway with predicted relevance across biomedical fields. Just as the cGAS-STING field has been driven forward by the hard work of virologists, structural biologists, biochemists, human geneticists, and immunologists, so we hope that the next decade will see diverse contributions from many scientists to enhance our understanding of the SIDSP and create a cohesive model of intracellular DNA sensing that will result in better treatments for human disease. 


\section{Chapter 6: Materials and methods}

\section{Reagent list}

\begin{tabular}{|c|c|c|c|}
\hline reagent type & designation & source or reference & identifier \\
\hline antibody & mouse monoclonal anti- $\beta$ Actin & SIGMA & cat \# A5441 \\
\hline antibody & rabbit monoloanl anti-ATM & \begin{tabular}{|l} 
Cell Signaling Technology \\
\end{tabular} & cat \#2873 \\
\hline antibody & rabbit polyclonal anti-DNA-PK & Cell Signaling Technology & cat \#4602 \\
\hline antibody & rabbit monoclonal anti-HSC70 & BioLegend & cat \# 682102 \\
\hline antibody & mouse monoclonal anti-HSV1 ICP0 & Santa Cruz & cat \# sc-53070 \\
\hline antibody & rabbit polyclonal anti-phospho H2AX (Ser 139) & Cell Signaling Technology & cat \#2577 \\
\hline antibody & rabbit monoclonal anti-phospho IRF3 (S386) & Abcam & cat \# ab76493 \\
\hline antibody & rabbit monoclonal anti-phospho IRF3 (S396) & Cell Signaling Technology & cat \# 4947 \\
\hline antibody & mouse monoclonal anti-Ku70 & Santa Cruz & cat \# sc-5309 \\
\hline antibody & rabbit monoclonal anti-Ku80 & Cell Signaling Technology & cat \#2180 \\
\hline antibody & rabbit monoclonal anti-LSD1 & Cell Signaling & cat \# 2184S \\
\hline antibody & rabbit monoclonal anti- STING & Cell Signaling Technology & cat \#13647 \\
\hline antibody & rabbit polyclonal anti- TBK1/NAK & Cell Signaling Technology & cat \#3013 \\
\hline antibody & rabbit monoclonal anti-phosho TBK1 (Ser172) & Cell Signaling Technology & cat \#5483 \\
\hline chemical & Calf Thymus DNA & SIGMA & cat \# D4764 \\
\hline chemical & 2'3'-cGAMP & Invivogen & cat \# tlrl-nacga23-1 \\
\hline chemical & Lipofectamine 2000 & Invitrogen & cat \#11668019 \\
\hline chemical & Nu-7441 (DNA-PK inhibitor) & SelleckChem & cat \# S2638 \\
\hline chemical & Ku-60019 (ATM inhibitor) & SelleckChem & cat \# S1570 \\
\hline chemical & Protease and Phosphatase inhibitor mini tablets & Thermo Fisher -- Pierce & cat \# PIA32961 \\
\hline chemical & Phorbol Myristoyl Acetate (PMA) & Sigma-Aldrich & cat\# P1585 \\
\hline chemical & PEI transfection reagent & Polysciences & cat \#23966-2 \\
\hline qPCR reagent & RNA to cDNA EcoDry Premix & Clonetech & cat \#639548 \\
\hline qPCR reagent & iTaq Universal SYBR Green Supermix & Bio-Rad & cat \#1725124 \\
\hline kit & $\begin{array}{l}\text { NEB HiScribe T7 High Yield RNA Synthesis } \\
\text { Kit }\end{array}$ & NEB & cat \# E2040S \\
\hline kit & Direct-zol RNA miniprep plus & Genessee Scientific & cat \#R2072 \\
\hline kit & Dynabeads ${ }^{\mathrm{TM}}$ Antibody Coupling Kit & ThermoFisher Scientific & cat \# 14311D \\
\hline kit & Zero blunt TOPO cloning kit & ThermoFisher Scientific & cat \#450245 \\
\hline filters & SteriFlip $0.45 \mathrm{mM}$ filters & Millipore & cat \# SE1M003M0 \\
\hline western reagent & BOLT $4-12 \%$ bis-tris plus gels & ThermoFisher Scientific & $\begin{array}{l}\text { cat \# } \\
\text { NW04125BOX }\end{array}$ \\
\hline western reagent & NuPAGE 3-8\% Tris-Acetate Protein Gels & ThermoFisher Scientific & cat \#EA03785BOX \\
\hline western reagent & Immobilon-FL PVDF, $0.45 \mu \mathrm{m}$ & SIGMA & cat \# IPFL00010 \\
\hline Plasmid & HA-human HSPA8 WT in pCDNA3 & this study & \\
\hline Plasmid & HA-human HSPA8 S637A in pCDNA3 & this study & \\
\hline Plasmid & HA-human HSPA8 S638A in pCDNA3 & this study & \\
\hline Plasmid & HA-human HSPA8 S637A/638A in pCDNA3 & this study & \\
\hline Plasmid & HA-mouse HSPA8 WT in pCDNA3 & this study & \\
\hline Plasmid & HA-mouse HSPA8 S637A/S638A in pCDNA3 & this study & \\
\hline Plasmid & ICP0 expression plasmid (p 110-CI) & Everett et al, 1999 & \\
\hline
\end{tabular}




\section{Ligands and inhibitors}

Sheared CT-DNA (Sigma) and 2'3' cGAMP (Invivogen) were purchased and diluted in

water; ISD oligos were ordered from Integrated DNA Technologies and annealed in water ${ }^{10}$; RIG-I ligand was synthesized in vitro as previously described ${ }^{99}$ using HiScribe T7 High Yield RNA Synthesis Kit. For plasmid stimulations, midiprepped pcDNA3 was either untreated or sonicated with a Covaris M220 focused ultrasonicator at 5\% ChIP (factory setting). Nu-7441 and Ku-60019 (SelleckChem) were suspended in DMSO and used to treat cells for 1 hour prior to stimulation with nucleic acid ligands. For Nu-7441, we used $0.25,0.5$, 1, or 2 uM. For Ku60019 , we used $0.125,0.25,0.5$, or $1 \mathrm{uM}$. Untreated cells received the same amounts of plain DMSO.

\section{Cell treatments}

HEK 293 cells were grown in DMEM supplemented with 10\% FCS, L-glutamine, penicillin/streptomycin, sodium pyruvate, and HEPES. U937 and THP1 cell lines were grown in RPMI supplemented as above, and differentiated prior to stimulation using $100 \mathrm{nM}$ phorbol myristoyl acetate (PMA) for 24 hours and then rested in media lacking PMA for 24 hours.

HEK 293 cells were plated at 0.5 million/well in a 6 well dish in $2 \mathrm{~mL}$ media the day before stimulation for protein harvest. For RNA harvest and qPCR, U937 cells were plated at 0.25 million/well in a 24 well dish. In the 6 well dish format, cells received 8 ug of CT DNA, ISD100, or pcDNA3 complexed with $8 \mathrm{ul}$ of Lipofectamine 2000. $10 \mathrm{uM}$ cGAMP was complexed with 8 ul Lipofectamine and 1 ug RIG-I ligand was complexed with 1 ul Lipofectamine to achieve comparable induction of IFN across treatments in competent cells. Stimulations done in 24 well plates were scaled by $1 / 4$. Etoposide (prepared in DMSO) was diluted in culture media to $50 \mu \mathrm{M}$, 
and untreated cells received the same volume of DMSO. Cells were irradiated with 30 Gy using a Rad Source RS 2000 X-irradiator.

\section{IFN bioassay}

Supernatants from stimulated cells were harvested 24 hours post-stimulation and used to stimulate a HeLa cell line stably expressing an ISRE-luciferase reporter as described previously ${ }^{58}$.

\section{Western blotting}

Cells were harvested by trypsinization (U937 cells) or vigorous wash with PBS (HEK293 cells), pelleted, and lysed using either a 1\% Triton-X-100 buffer (20 mM HEPES, $150 \mathrm{mM}$ $\mathrm{NaCl}, 10 \%$ glycerol, $1 \mathrm{mM}$ EDTA, Pierce phosphatase/protease inhibitors) or, for samples requiring measurement of DNA-PK protein levels, RIPA buffer (150 mM NaCl, $1 \%$ Triton-X100, $0.5 \%$ sodium deoxycholate, $0.1 \%$ SDS, $50 \mathrm{mM}$ Tris $\mathrm{pH}$ 8.0, Pierce phosphatase/protease

inhibitors). Lysates were vortexed and incubated on ice for 15 minutes before clearing by centrifugation for 15 minutes. Proteins were separated on Bolt 4-12\% Bis-Tris gels (ThermoFisher) in MES buffer for 30 minutes at $200 \mathrm{~V}$ and transferred to Immobilon-FL PVDF membrane (Sigma). Blots were blocked in 5\% BSA/TBST for 30 minutes prior to incubation with primary. The pIRF3 S386 blots were incubated at $4^{0} \mathrm{C}$ overnight and washed at least 30 minutes in TBST prior to secondary incubation to prevent background. In order to better resolve DNA-PK (470 kDa), lysates were run on 3-8\% Tris-acetate gels (ThermoFisher) for 2 hours at $150 \mathrm{~V}$ and then transferred in $5 \%$ methanol for 3 hours at $20 \mathrm{~V}$ at $4^{\circ} \mathrm{C}$.

\section{LentiCRISPR targeting}

VSV-G pseudotyped, self-inactivating lentivirus was prepared by transfecting a $60-80 \%$ confluent $10-\mathrm{cm}$ plate of HEK $293 \mathrm{~T}$ cells with $1.5 \mathrm{ug}$ of pVSV-G expression vector, 3 ug of 
pMDLg/pRRE, 3 ug pRSV-Rev and 6 ug of pRRL lentiCRISPR vectors using Poly(ethyleneimine) (PEI; Sigma). Media was replaced 24 hours post-transfection and harvested 24 hours later for filtration with a 0.45 um filter (SteriFlip, Millipore). Approximately 1 million cells were transduced with $10 \mathrm{~mL}$ filtered virus. Targeting NHEJ components efficiently was difficult; best results were achieved by increasing transduction rates with sequential transductions on two consecutive days. Cells were plated for stimulations while still under selection at day 4 post first transduction.

For CRISPR/Cas9 gene targeting, we generated pRRL lentiviral vectors in which a U6 promoter drives expression of a gRNA, and an MND promoter drives expression of Cas9, a T2A peptide, and either a puromycin or blasticidin ${ }^{19}$. gRNA sequences are as follows, where the (G) denotes a nucleotide added to enable robust transcription off the U6 promoter and the underlined sequence denotes the Protospacer Adjacent Motif (PAM):

H1 non-targeting control: (G)ACGGAGGCTAAGCGTCGCAA ${ }^{70}$; TMEM173 (STING): GGTGCCTGATAACCTGAGTATGG;

TBK1: (G)CATAAGCTTCCTTCGTCCAGTGG;

PRKDC (DNA-PK): GCAGGAGACCTTGTCCGCTGCGG; XRCC6 (Ku70): GATCCGTGGCCCATCATGTCTTGG; XRCC5(Ku80):GTTGTGCTGTGTATGGACGTGGG;

ATM guide 1: (G)CCAAGGCTATTCAGTGTGCG $\underline{A G G}^{70}$; ATM guide 2: (G)TGATAGAGCTACAGAACGAAAGG;

E1A: (G)AAGACCTGCAACCGTGCCCGGGG ${ }^{58}$.

Guides against $P R K D C, X R C C 6$, and $X R C C 5$ were designed using Benchling.

\section{Generation of clonal cell lines}


KO cell lines were generated by limiting dilution, screened by western blot, and verified by Sanger sequencing and functional assays. The STING/DNA-PK DKO U937 cell line was produced by transducing U937s simultaneously with a STING lentiCRISPR puro virus and a DNA-PK lentiCRISPR blasticidin virus, selecting in $10 \mathrm{ug} / \mathrm{ml}$ puro and $5 \mathrm{ug} / \mathrm{ml}$ blasticidin, and seeding in 96 well plates immediately after selection. Very few colonies grew, and the verified DKO clone grew markedly slower than $\mathrm{H} 1$ non-targeted control clones or the STING KO clones, as expected ${ }^{73}$.

PCR primers used for amplifying genomic DNA surrounding CRISPR targeting sites in clonal lines were as follows (Forward/Reverse):

TMEM173: 5'-AGCTCCAGGCCCGGATTCG-3'/5'-TGCCCGTTCTCCAGAAGCTC-3'

TBK1: 5'- CCCTACTGTATCCTCATG-3'/5'-CTTACCTCCTCTTCAATAGC-3'

PRKDC: 5'-GGGGCATTTCCGGGTCCGGG-3'/5'-TGCCCTGCCCCCCACTCTGC-3'

Amplicons were cloned using the Zero Blunt TOPO PCR Cloning kit (ThermoFisher), prepared as plasmids, and then several individual plasmids were sequenced. Sequencing alignments were made using Benchling.

\section{RNA isolation and qPCR}

Cells were harvested in Trizol before purification via Direct-zol RNA miniprep (Genesee Scientific) per manufacturer's instructions with an additional dry spin after disposing of the final wash to prevent carryover. cDNA was generated using EcoDry double primed premix (Clontech). qPCR was performed using iTaq supermix on the Bio-Rad CFX96 Real-Time system. Human gene PCR primer sequences are as follows:

GAPDH Fwd: 5'- AACAGCCTCAAGATCATCAGC-3', GAPDH Rev: 5'-CACCACCTTCTTGATGTCATC-3' 
IFNB1 Fwd: 5'-ACGCCGCATTGACCATCTATG-3',

IFNB1 Rev: 5'-CGGAGGTAACCTGTAAGTCTGT-3'.

Mouse primer sequences are as follows:

Hprt Fwd: 5'-GTTGGATACAGGCCAGATTTGTTG-3',

Hprt Rev: 5'-GAGGGTAGGCTGGCCTATAGGCT-3'

Ifnb Fwd: 5'-GCACTGGGTGGAATGAGACTATTG-3'

Ifnb Rev: 5'-TTCTGAGGCATCAACTGACAGGTC -3'.

mRNA-Seq and analysis

Total RNA was added directly to lysis buffer from the SMART-Seq v4 Ultra Low Input RNA Kit for Sequencing (Takara), and reverse transcription was performed followed by PCR amplification to generate full length amplified cDNA. Sequencing libraries were constructed using the NexteraXT DNA sample preparation kit (Illumina) to generate Illumina-compatible barcoded libraries. Libraries were pooled and quantified using a Qubit ${ }^{\circledR}$ Fluorometer (Life Technologies). Dual-index, single-read sequencing of pooled libraries was carried out on a HiSeq2500 sequencer (Illumina) with 58-base reads, using HiSeq v4 Cluster and SBS kits (Illumina) with a target depth of 5 million reads per sample. Base calls were processed to FASTQs on BaseSpace (Illumina), and a base call quality trimming step was applied to remove low-confidence base calls from the ends of reads. The FASTQs were aligned to the human reference genome using the STAR aligner, and gene counts were generated using htseq-count.

Exploratory analysis and statistics were run using R (version 3.5.1) and bioconductor (version 3.7). The gene count matrix was filtered by a row mean of ten or greater counts and normalized with EDGER. LogCPM transformation was performed using voom through the 
limma bioconductor package (3.34.8). Statistical analysis (including differential expression) was performed using the limma package $\mathrm{e}^{100,101}$.

Co-expression was performed on genes statistically significant in the differential expression analysis (threshold: linear fold change $>=|1.5|$ and FDR $<=0.05$ ) in at least one comparison. The union of these DE genes were loaded into R and filtered by known interferon signaling genes using all of the GO terms listed in Table S1. Correlations (ward.2 clustering and euclidean distance) were run on the union of $\log 2 \mathrm{FC}$ using the WGCNA and heatmap.2 bioconductor packages in $\mathrm{R}^{100,102,103}$.

\section{Immunoprecipitation and Mass Spectrometry}

To identify HSPA8 using mass spectrometry, we performed immunoprecipitation of CTDNA stimulated HEK293 cells using the antibody to IRF3 pS386 crosslinked to Dynabeads (ThermoFisher) overnight at $4 \mathrm{C}$, then washed three times in lysis buffer and two times in ammonium biocarbonate $(50 \mathrm{mM})$ before peptide digestion (V5280, Promega). Peptides were loaded onto a 3-cm self-packed C18 capillary pre-column (Reprosil $5 \mu \mathrm{M}$, Dr. Maisch). After a 10 -min rinse ( $0.1 \%$ Formic Acid), the pre-column was connected to a $25-\mathrm{cm}$ self-packed $\mathrm{C} 18$ (Reliasil $3 \mu \mathrm{M}$, Orochem) analytical capillary column (inner diameter, 50- $\mu \mathrm{m}$; outer diameter, $360-\mu \mathrm{m})$ with an integrated electrospray tip $(\sim 1-\mu \mathrm{m}$ orifice). Online peptide separation followed by mass spectrometric analyses was performed on a 2D-nanoLC system (nanoAcquity UPLC system, Waters Corp.). Peptides were eluted using a 150-min gradient with solvent A $\left(\mathrm{H}_{2} \mathrm{O} /\right.$ Formic Acid, 99.9:1 (v/v)) and B (Acetonitrile/Formic Acid, 99.9:1 (v/v)): 10 min from $0 \%$ to $10 \% \mathrm{~B}, 105 \mathrm{~min}$ from $10 \%$ to $40 \% \mathrm{~B}, 15 \mathrm{~min}$ from $40 \%$ to $80 \% \mathrm{~B}$, and 20 minutes with 100\% A. Eluted peptides were directly electrosprayed into a Orbitrap QExactive mass spectrometer (Thermo Fisher Scientific) equipped with a high energy collision cell (HCD). The 
mass spectrometer was operated in a data-dependent mode to automatically switch between MS and MS/MS acquisitions. Each full scan (from $m / z$ 300-1500) was acquired in the Orbitrap analyzer (resolution $=70,000$ ), followed by MS/MS analyses on the top twenty most intense precursor ions that had charge states greater than one. The HCD MS/MS scans were acquired using the Orbitrap system (resolution $=17,500$ ) at normalized collision energy of $28 \%$. The precursor isolation width was set at $2 \mathrm{~m} / \mathrm{z}$ for each MS/MS scan and the maximum ion accumulation times were as follows: MS (100ms), MS/MS (100ms). MS/MS data files were searched using the Comet algorithm ${ }^{104}$, and the data were further processed using the Institute for System’s Biology's Trans-Proteomic Pipeline ${ }^{105}$. Static modification of cysteine (carbamidomethylation; 57.02 Da) was used in the search.

\section{Cloning of HSPA8}

PCR and InFusion cloning (Clonetech) were used to generate N-terminal HA-tagged WT and alanine mutant human HSPA8 constructs from HEK 293 cell cDNA. A murine HSPA8 cDNA clone (Transomic technologies, Clone ID BC089322) was used as template to generate the epitope-tagged mouse versions.

\section{Transient expression of ICP0}

0.25 million STING KO HEK293s were seeded in 12 well format the day before transfection with $0,1,2$ or 4 ug of ICP0 expression plasmid using Lipofectamine 2000 at a 1 ul:1 ug DNA ratio. Empty pcDNA3 was used to bring the total amount of transfected DNA up to 4 ug

total. 24 hours post-transfection, the cells were treated with 4 ug CT DNA or 4 ul Lipofectamine 2000 alone and harvested 3 hours later in RIPA buffer with phosphatase inhibitors for analysis by western blot. 


\section{Experimental replicates and statistics}

All experiments presented in this study, except the mRNA-Seq studies, were done two or more times, with biological triplicates for each condition in RT-qPCR experiments. Quantitative data were visualized and analyzed using GraphPad Prism software. Multiple unpaired t-tests with significance determined by Holm-Sidak method were used to compare differences between groups, unless otherwise noted for specific tests in figure legends. Significance is indicated as follows: ${ }^{*} \mathrm{p}<0.05 . * * \mathrm{p}<0.01, * * * \mathrm{p}<0.001, * * * * \mathrm{p}<0.0001$. 


\section{References}

1. Schneider, W. M., Chevillotte, M. D. \& Rice, C. M. Interferon-stimulated genes: a complex web of host defenses. Annu. Rev. Immunol. 32, 513-45 (2014).

2. Muller, U., Steinhoff, U., Reis, L. F. \& Aguet, M. Functional role of type I and type II interferons in antiviral defense. Science (80-. ). 264, 1918-1921 (1994).

3. Kolumam, G. A., Thomas, S., Thompson, L. J., Sprent, J. \& Murali-Krishna, K. Type I interferons act directly on CD8 T cells to allow clonal expansion and memory formation in response to viral infection. J. Exp. Med. 202, 637-50 (2005).

4. Le Bon, A. et al. Cross-priming of CD8+ T cells stimulated by virus-induced type I interferon. Nat. Immunol. 4, 1009-1015 (2003).

5. Pandey, S., Kawai, T. \& Akira, S. Microbial sensing by Toll-like receptors and intracellular nucleic acid sensors. Cold Spring Harb. Perspect. Biol. 7, a016246 (2014).

6. Diebold, S. S. et al. Viral infection switches non-plasmacytoid dendritic cells into high interferon producers. Nature 424, 324-328 (2003).

7. Yoneyama, M. et al. The RNA helicase RIG-I has an essential function in double-stranded RNA-induced innate antiviral responses. Nat. Immunol. 5, 730-737 (2004).

8. Yoneyama, M. et al. Shared and unique functions of the DExD/H-box helicases RIG-I, MDA5, and LGP2 in antiviral innate immunity. J. Immunol. 175, 2851-8 (2005).

9. Kawai, T. et al. IPS-1, an adaptor triggering RIG-I- and Mda5-mediated type I interferon induction. Nat. Immunol. 6, 981-988 (2005).

10. Stetson, D. B. \& Medzhitov, R. Recognition of cytosolic DNA activates an IRF3dependent innate immune response. Immunity 24, 93-103 (2006).

11. Zhong, B. et al. The Adaptor Protein MITA Links Virus-Sensing Receptors to IRF3 Transcription Factor Activation. Immunity 29, 538-550 (2008).

12. Ishikawa, H. \& Barber, G. N. STING is an endoplasmic reticulum adaptor that facilitates innate immune signalling. Nature 455, 674-8 (2008).

13. Ishikawa, H., Ma, Z. \& Barber, G. N. STING regulates intracellular DNA-mediated, type I interferon-dependent innate immunity. Nature 461, 788-92 (2009).

14. Takaoka, A. et al. DAI (DLM-1/ZBP1) is a cytosolic DNA sensor and an activator of innate immune response. Nature 448, 501-505 (2007).

15. Ishii, K. J. et al. TANK-binding kinase- 1 delineates innate and adaptive immune responses to DNA vaccines. Nature 451, 725-729 (2008).

16. Ablasser, A. et al. RIG-I-dependent sensing of poly(dA:dT) through the induction of an RNA polymerase III-transcribed RNA intermediate. Nat. Immunol. 10, 1065-1072 (2009).

17. Zhang, Z. et al. The helicase DDX41 senses intracellular DNA mediated by the adaptor STING in dendritic cells. Nat. Immunol. 12, 959-965 (2011).

18. Brunette, R. L. et al. Extensive evolutionary and functional diversity among mammalian AIM2-like receptors. J. Exp. Med. 209, 1969-83 (2012).

19. Gray, E. E. et al. The AIM2-like Receptors Are Dispensable for the Interferon Response to Intracellular DNA. Immunity 45, 1-12 (2016).

20. Unterholzner, L. et al. IFI16 is an innate immune sensor for intracellular DNA. Nat. Immunol. 11, 997-1004 (2010).

21. Sun, L., Wu, J., Du, F., Chen, X. \& Chen, Z. J. Cyclic GMP-AMP Synthase Is a Cytosolic DNA Sensor That Activates the Type I Interferon Pathway. Science (80-. ). 339, 786-791 (2013). 
22. Wu, J., Sun, L. \& Zhijian J., C. Cyclic GMP-AMP Is an Endogenous Second Messenger in Innate Immune Signaling by Cytosolic DNA. Science (80-. ). 339, 826-830 (2013).

23. Burdette, D. L. et al. STING is a direct innate immune sensor of cyclic di-GMP. Nature 478, 515-518 (2011).

24. Ablasser, A. et al. cGAS produces a 2'-5'-linked cyclic dinucleotide second messenger that activates STING. Nature 498, 380-4 (2013).

25. Gao, D. et al. Cyclic GMP-AMP synthase is an innate immune sensor of HIV and other retroviruses. Science 341, 903-6 (2013).

26. Almine, J. F. et al. IFI16 and cGAS cooperate in the activation of STING during DNA sensing in human keratinocytes. Nat. Commun. 8, 14392 (2017).

27. Jønsson, K. L. et al. IFI16 is required for DNA sensing in human macrophages by promoting production and function of cGAMP. Nat. Commun. 8, 14391 (2017).

28. Gao, P. et al. Structure-function analysis of STING activation by c[G(2',5')pA(3',5')p] and targeting by antiviral DMXAA. Cell 154, 748-62 (2013).

29. Shu, C. et al. Structural Insights into the Functions of TBK1 in Innate Antimicrobial Immunity. 72, 181-204 (2013).

30. Liu, S. et al. Phosphorylation of innate immune adaptor proteins MAVS, STING, and TRIF induces IRF3 activation. Science 347, aaa2630-1-aaa2630-13 (2015).

31. Hu, M.-M. et al. Sumoylation Promotes the Stability of the DNA Sensor cGAS and the Adaptor STING to Regulate the Kinetics of Response to DNA Virus. Immunity 45, 555569 (2016).

32. Lin, R., Heylbroeck, C., Pitha, P. M. \& Hiscott, J. Virus-Dependent Phosphorylation of the IRF-3 Transcription Factor Regulates Nuclear Translocation, Transactivation Potential, and Proteasome-Mediated Degradation. Mol. Cell. Biol. 18, 2986-2996 (1998).

33. Servant, M. J. et al. Identification of the minimal phosphoacceptor site required for in vivo activation of interferon regulatory factor 3 in response to virus and double-stranded RNA. J. Biol. Chem. 278, 9441-7 (2003).

34. Mori, M. et al. Identification of Ser-386 of interferon regulatory factor 3 as critical target for inducible phosphorylation that determines activation. J. Biol. Chem. 279, 9698-702 (2004).

35. Li, X.-D. et al. Pivotal Roles of cGAS-cGAMP Signaling in Antiviral Defense and Immune Adjuvant Effects. 120, 1390-1394 (2013).

36. Lam, E., Stein, S. \& Falck-Pedersen, E. Adenovirus detection by the cGAS/STING/TBK1 DNA sensing cascade. J. Virol. 88, 974-81 (2014).

37. Lahaye, X. et al. NONO Detects the Nuclear HIV Capsid to Promote cGAS-Mediated Innate Immune Activation. Cell 175, 488-501.e22 (2018).

38. Volkman, H. E., Cambier, S., Gray, E. E. \& Stetson, D. B. cGAS is predominantly a nuclear protein. bioRxiv 486118 (2018). doi:10.1101/486118

39. Härtlova, A. et al. DNA Damage Primes the Type I Interferon System via the Cytosolic DNA Sensor STING to Promote Anti-Microbial Innate Immunity. Immunity 42, 332-343 (2015).

40. Heijink, A. M. et al. BRCA2 deficiency instigates cGAS-mediated inflammatory signaling and confers sensitivity to tumor necrosis factor-alpha-mediated cytotoxicity. Nat.

Commun. 10, 100 (2019).

41. Glück, S. et al. Innate immune sensing of cytosolic chromatin fragments through cGAS promotes senescence. Nat. Cell Biol. 19, 1061-1070 (2017). 
42. Yang, H., Wang, H., Ren, J., Chen, Q. \& Chen, Z. J. cGAS is essential for cellular senescence. Proc. Natl. Acad. Sci. U. S. A. 114, E4612-E4620 (2017).

43. Dou, Z. et al. Cytoplasmic chromatin triggers inflammation in senescence and cancer. Nature 550, 402-406 (2017).

44. Blackford, A. N. \& Jackson, S. P. ATM, ATR, and DNA-PK: The Trinity at the Heart of the DNA Damage Response. Mol. Cell 66, 801-817 (2017).

45. Kondo, T. et al. DNA damage sensor MRE11 recognizes cytosolic double-stranded DNA and induces type I interferon by regulating STING trafficking. Proc. Natl. Acad. Sci. U.S. A. 110, 2969-74 (2013).

46. Härtlova, A. et al. DNA Damage Primes the Type I Interferon System via the Cytosolic DNA Sensor STING to Promote Anti-Microbial Innate Immunity. Immunity 42, 332-343 (2015).

47. Ferguson, B. J., Mansur, D. S., Peters, N. E., Ren, H. \& Smith, G. L. DNA-PK is a DNA sensor for IRF-3-dependent innate immunity. Elife 1, e00047 (2012).

48. Karpova, A. Y., Trost, M., Murray, J. M., Cantley, L. C. \& Howley, P. M. Interferon regulatory factor-3 is an of DNA-PK in vivo target. Proc Natl Acad Sci U S A 99, 28182823 (2002).

49. Beamish, H. J. et al. The C-terminal conserved domain of DNA-PKcs, missing in the SCID mouse, is required for kinase activity. Nucleic Acids Res. 28, 1506-13 (2000).

50. Peters, N. E. et al. A Mechanism for the Inhibition of DNA-PK-Mediated DNA Sensing by a Virus. PLoS Pathog. 9, (2013).

51. Orzalli, M. H. \& Knipe, D. M. Cellular Sensing of Viral DNA and Viral Evasion Mechanisms. Annu. Rev. Microbiol. 477-492 (2014). doi:10.1146/annurev-micro-091313103409

52. Morchikh, M. et al. HEXIM1 and NEAT1 Long Non-coding RNA Form a Multi-subunit Complex that Regulates DNA-Mediated Innate Immune Response. Mol. Cell 67, 387399.e5 (2017).

53. Hancks, D. C., Hartley, M. K., Hagan, C., Clark, N. L. \& Elde, N. C. Overlapping Patterns of Rapid Evolution in the Nucleic Acid Sensors cGAS and OAS1 Suggest a Common Mechanism of Pathogen Antagonism and Escape. PLOS Genet. 11, e1005203 (2015).

54. Mozzi, a. et al. OASes and STING: adaptive evolution in concert. Genome Biol. Evol. 7, 1016-1032 (2015).

55. Su, C. \& Zheng, C. Herpes Simplex Virus 1 Abrogates the cGAS/STING-Mediated Cytosolic DNA-Sensing Pathway via Its Virion Host Shutoff Protein, UL41. J. Virol. 91, e02414-16 (2017).

56. Zhang, J. et al. Species-Specific Deamidation of cGAS by Herpes Simplex Virus UL37 Protein Facilitates Viral Replication. Cell Host Microbe 24, 234-248.e5 (2018).

57. Huang, J. et al. Herpes Simplex Virus 1 Tegument Protein VP22 Abrogates cGAS/STING-Mediated Antiviral Innate Immunity. J. Virol. 92, (2018).

58. Lau, L., Gray, E. E., Brunette, R. L. \& Stetson, D. B. DNA tumor virus oncogenes antagonize the cGAS-STING DNA-sensing pathway. Science (80-. ). 350, 568-571 (2015).

59. Huang, Z.-F. et al. Human Cytomegalovirus Protein UL31 Inhibits DNA Sensing of cGAS to Mediate Immune Evasion. Cell Host Microbe 24, 69-80 (2018).

60. Zhang, G. et al. Cytoplasmic isoforms of Kaposi sarcoma herpesvirus LANA recruit and antagonize the innate immune DNA sensor cGAS. (2015). doi:10.1073/pnas.1516812113 
61. Ma, Z. et al. Modulation of the cGAS-STING DNA sensing pathway by gammaherpesviruses. Proc. Natl. Acad. Sci. U. S. A. 112, E4306-15 (2015).

62. Wu, J. et al. Inhibition of cGAS DNA Sensing by a Herpesvirus Virion Protein. Cell Host Microbe 1-12 (2015). doi:10.1016/j.chom.2015.07.015

63. Liu, Y. et al. Hepatitis B Virus Polymerase Disrupts K63-Linked Ubiquitination of STING To Block Innate Cytosolic DNA-Sensing Pathways. J. Virol. 89, 2287-2300 (2015).

64. Aguirre, S. et al. Dengue virus NS2B protein targets cGAS for degradation and prevents mitochondrial DNA sensing during infection. Nat. Microbiol. 2, 17037 (2017).

65. Lin, R. et al. HHV-8 encoded vIRF-1 represses the interferon antiviral response by blocking IRF-3 recruitment of the CBP/p300 coactivators. Oncogene. 20, 800-811. (2001).

66. Ma, Z. et al. Modulation of the cGAS-STING DNA sensing pathway by gammaherpesviruses. Proc. Natl. Acad. Sci. E4306-E4315 (2015).

doi:10.1073/pnas.1503831112

67. DeCaprio, J. a. How the Rb tumor suppressor structure and function was revealed by the study of Adenovirus and SV40. Virology 384, 274-284 (2009).

68. Louis, N., Evelegh, C. \& Graham, F. L. Cloning and Sequencing of the Cellular-Viral Junctions from the Human Adenovirus Type 5 Transformed 293 Cell Line. Virology 233, 423-429 (1997).

69. Graham, F. L., Smileyt, J., Russell, W. C. \& Nairn, R. Characteristics of a Human Cell Line Transformed by DNA from Human Adenovirus Type 5. J. gen. Virol 36, (2977).

70. Sanjana, N. E., Shalem, O. \& Zhang, F. Improved vectors and genome-wide libraries for CRISPR screening. Nat. Methods 11, 006726 (2014).

71. Golding, S. E. et al. Improved ATM kinase inhibitor KU-60019 radiosensitizes glioma cells, compromises insulin, AKT and ERK prosurvival signaling, and inhibits migration and invasion. Mol. Cancer Ther. 8, 2894-2902 (2009).

72. Leahy, J. J. J. et al. Identification of a highly potent and selective DNA-dependent protein kinase (DNA-PK) inhibitor (NU7441) by screening of chromenone libraries. Bioorg. Med. Chem. Lett. 14, 6083-6087 (2004).

73. Ruis, B. L., Fattah, K. R. \& Hendrickson, E. A. The catalytic subunit of DNA-dependent protein kinase regulates proliferation, telomere length, and genomic stability in human somatic cells. Mol. Cell. Biol. 28, 6182-95 (2008).

74. Fattah, F. J., Lichter, N. F., Fattah, K. R., Oh, S. \& Hendrickson, E. A. Ku70, an essential gene, modulates the frequency of rAAV-mediated gene targeting in human somatic cells. Proc. Natl. Acad. Sci. 105, 8703-8708 (2008).

75. Li, G., Nelsen, C. \& Hendrickson, E. A. Ku86 is essential in human somatic cells. Proc. Natl. Acad. Sci. U. S. A. 99, 832-7 (2002).

76. Conlon, J. et al. Mouse, but not human STING, binds and signals in response to the vascular disrupting agent 5,6-dimethylxanthenone-4-acetic acid. J. Immunol. 190, 521625 (2013).

77. Finnie, N. J., Gottlieb, T. M., Blunt, T., Jeggo, P. a \& Jackson, S. P. DNA-dependent protein kinase activity is absent in xrs-6 cells: implications for site-specific recombination and DNA double-strand break repair. Proc. Natl. Acad. Sci. U. S. A. 92, 320-324 (1995).

78. Jette, N. \& Lees-Miller, S. P. The DNA-dependent protein kinase: A multifunctional protein kinase with roles in DNA double strand break repair and mitosis. Prog. Biophys. 
Mol. Biol. 117, 194-205 (2015).

79. Neal, J. A., Xu, Y., Abe, M., Hendrickson, E. \& Meek, K. Restoration of ATM Expression in DNA-PKcs-Deficient Cells Inhibits Signal End Joining. J. Immunol. 196, 3032-42 (2016).

80. Parkinson, J., Lees-Miller, S. P. \& Everett, R. D. Herpes simplex virus type 1 immediateearly protein vmw110 induces the proteasome-dependent degradation of the catalytic subunit of DNA-dependent protein kinase. J. Virol. 73, 650-7 (1999).

81. Moss, S. M. et al. A Legionella pneumophila Kinase Phosphorylates the Hsp70 Chaperone Family to Inhibit Eukaryotic Protein Synthesis. Cell Host Microbe 25, 454 462.e6 (2019).

82. Corrales, L. et al. Direct Activation of STING in the Tumor Microenvironment Leads to Potent and Systemic Tumor Regression and Immunity. Cell Rep. 11, 1018-1030 (2015).

83. Sivick, K. E. et al. Magnitude of Therapeutic STING Activation Determines CD8+ T Cell-Mediated Anti-tumor Immunity. Cell Rep. 25, 3074-3085.e5 (2018).

84. Xia, T. et al. Deregulation of STING Signaling in Colorectal Carcinoma Constrains DNA Damage Responses and Correlates With Tumorigenesis. CellReports 1-16 (2016). doi:10.1016/j.celrep.2015.12.029

85. Gray, E. E., Treuting, P. M., Woodward, J. J. \& Stetson, D. B. Cutting Edge: cGAS Is Required for Lethal Autoimmune Disease in the Trex1-Deficient Mouse Model of Aicardi-Goutières Syndrome. J. Immunol. 195, 1939-43 (2015).

86. König, N. et al. Familial chilblain lupus due to a gain-of-function mutation in STING. Ann. Rheum. Dis. 76, 468-472 (2017).

87. Liu, Y. et al. Activated STING in a Vascular and Pulmonary Syndrome. N. Engl. J. Med. 1-12 (2014). doi:10.1056/NEJMoa1312625

88. Turnell, A. S. \& Grand, R. J. DNA viruses and the cellular DNA-damage response. J. Gen. Virol. 93, 2076-97 (2012).

89. Stracker, T. H., Carson, C. T. \& Weitzman, M. D. Adenovirus oncoproteins inactivate the Mre11-Rad50-NBS1 DNA repair complex. Nature 418, 348-352 (2002).

90. Boyer, J., Rohleder, K. \& Ketner, G. Adenovirus E4 34k and E4 11k Inhibit Double Strand Break Repair and Are Physically Associated with the Cellular DNA-Dependent Protein Kinase. Virology 263, 307-312 (1999).

91. Frost, J. R. et al. The interaction of adenovirus E1A with the mammalian protein Ku70/XRCC6. Virology 500, 11-21 (2017).

92. King, C. R., Zhang, A., Tessier, T. M., Gameiro, S. F. \& Mymryk, J. S. Hacking the Cell: Network Intrusion and Exploitation by Adenovirus E1A. (2018).

doi:10.1128/mBio.00390-18

93. Fonseca, G. J. et al. Adenovirus evasion of interferon-mediated innate immunity by direct antagonism of a cellular histone posttranslational modification. Cell Host Microbe 11, 597-606 (2012).

94. Frost, J. R. et al. The interaction of adenovirus E1A with the mammalian protein Ku70/XRCC6. Virology 500, 11-21 (2017).

95. Muylaert, I. \& Elias, P. Knockdown of DNA ligase IV/XRCC4 by RNA interference inhibits herpes simplex virus type I DNA replication. J. Biol. Chem. 282, 10865-72 (2007).

96. Smith, S., Reuven, N., Mohni, K. N., Schumacher, A. J. \& Weller, S. K. Structure of the Herpes Simplex Virus 1 Genome: Manipulation of Nicks and Gaps Can Abrogate 
Infectivity and Alter the Cellular DNA Damage Response. J. Virol. 88, 10146-10156 (2014).

97. Hammarsten, O., DeFazio, L. G. \& Chu, G. Activation of DNA-dependent protein kinase by single-stranded DNA ends. J. Biol. Chem. 275, 1541-50 (2000).

98. Lin, R., Noyce, R. S., Collins, S. E., Everett, R. D. \& Mossman, K. L. The herpes simplex virus ICP0 RING finger domain inhibits IRF3- and IRF7-mediated activation of interferon-stimulated genes. J. Virol. 78, 1675-84 (2004).

99. Saito, T., Owen, D. M., Jiang, F., Marcotrigiano, J. \& Gale, M. Innate immunity induced by composition-dependent RIG-I recognition of hepatitis C virus RNA. Nature 454, 523527 (2008).

100. Smyth, G. Stat Appl Genet Mol Biol. Linear models and empirical Bayes methods for assessing differential expression in microarray experiments. (2004).

101. Law, C. W., Chen, Y., Shi, W. \& Smyth, G. K. voom: precision weights unlock linear model analysis tools for RNA-seq read counts. Genome Biol. 15, R29 (2014).

102. Loraine, A. E. et al. Analysis and Visualization of RNA-Seq Expression Data Using RStudio, Bioconductor, and Integrated Genome Browser. in 481-501 (Humana Press, New York, NY, 2015). doi:10.1007/978-1-4939-2444-8_24

103. Gentleman, R. C. et al. Bioconductor: open software development for computational biology and bioinformatics. Genome Biol. 5, R80 (2004).

104. Eng, J. K., Jahan, T. A. \& Hoopmann, M. R. Comet: An open-source MS/MS sequence database search tool. Proteomics 13, 22-24 (2013).

105. Keller, A., Eng, J., Zhang, N., Li, X. \& Aebersold, R. A uniform proteomics MS/MS analysis platform utilizing open XML file formats. Mol. Syst. Biol. 1, 2005.0017 (2005). 